\title{
Dorsolateral Striatal Selection and Frontal Cortex Inhibition for a Selective Detection Task in Mice
}

Running title: Striatal Selection and Cortical Inhibition for Selective Detection

Authors: Behzad Zareian ${ }^{1}$, Angelina Lam ${ }^{2}$, Zhaoran Zhang ${ }^{3}$, Edward Zagha ${ }^{1,3}$

${ }^{1}$ Department of Psychology, ${ }^{2}$ School of Medicine Division of Biomedical Sciences,

${ }^{3}$ Neuroscience Graduate Program, University of California Riverside, 900 University

Avenue, Riverside CA 92521 USA

Correspondence to: edward.zagha@ucr.edu 


\begin{abstract}
A learned sensory-motor behavior engages multiple brain regions, including the neocortex and the basal ganglia. How these brain regions coordinate to affect sensory selection (for target stimuli) and inhibition (for distractor stimuli) remains unknown. Here, we performed laminar electrophysiological recordings and muscimol inactivation in frontal cortex and dorsolateral striatum to determine the representations within and functions of each region during selective detection performance. From the recording experiments, in deep layers of frontal cortex and dorsolateral striatum we observed similar encoding of sensory and motor features. However, muscimol inactivation of each region resulted in drastically different outcomes. Inactivation of target-aligned striatum substantially reduced behavioral responses to all task stimuli without disrupting the ability to respond, suggesting essential roles in sensory selection. In contrast, inactivation of distractor-aligned frontal cortex increased responses to distractor stimuli, indicating essential roles in distractor inhibition. Overall, these data suggest distinct functions of frontal cortex and dorsolateral striatum in this task, despite having similar neuronal representations.
\end{abstract}

\title{
Keywords
}

Neocortex, striatum, inactivation, behavioral inhibition, sensory selection 


\section{Introduction}

Goal-directed behavior requires the ability to selectively respond to relevant sensory stimuli (selection) while inhibiting responses to extraneous stimuli (inhibition). Sensory selection and inhibition are fundamental cognitive processes that are highly relevant in everyday life and are compromised in a range of neurologic and psychiatric disorders (Avanzino et al., 2018; Baranek et al., 2013; Ford et al., 2009). While many brain structures interact to carry out goal-directed behaviors, the localization and distribution of various cognitive processes such as selection and inhibition continues to be heavily debated (Aron et al., 2014; Cools et al., 2004; Pessoa, 2014; Redgrave et al., 1999).

Formative neuronal recording studies within the neocortex have demonstrated full sensory-motor transformations unfolding across cortex, from stimulus encoding, to selection and attenuation, to motor triggering (de Lafuente \& Romo, 2006; Hanes \& Schall, 1996; Moran \& Desimone, 1985; Salinas \& Romo, 1998; Siegel et al., 2015). These studies provided strong motivation for considering neocortex as the primary structure implementing selective, goaldirected behaviors. Simultaneously, formative anatomical, physiological, and lesioning studies of the basal ganglia have identified these subcortical structures as essential for implementing action selection and initiation (Alexander \& Crutcher, 1990; Bergstrom et al., 2020; Bergstrom et al., 2018; Graybiel et al., 1994; Grillner et al., 2005; Jin \& Costa, 2010; Lee et al., 2019; Peters et al., 2021; Stephenson-Jones et al., 2011; Yin et al., 2004). Frontal cortex and dorsolateral striatum, in particular, are heavily interconnected. Therefore, it is quite possible that cognitive processes such as selection and inhibition are distributed functions of both neural structures (collectively referred to as the 'frontostriatal network') (Aron et al., 2014; Owen et al., 1996; Peters et al., 2021). Alternatively, frontal cortex and dorsolateral striatum may implement specialized, non- 
redundant functions during goal-directed tasks (Cools et al., 2004; Jin \& Costa, 2010; Partiot et al., 1996). Distinguishing between these possibilities requires performing representational and causal studies from both structures in subjects performing the same behavioral task. However, such studies, particularly in the context of selection and inhibition, have not been performed to date.

The mouse whisker system is an ideal model system to test these competing hypotheses, due to its simplified neural anatomy. Whisker stimulus responses propagate to the contralateral whisker representation of primary somatosensory cortex (wS1). In turn, wS1 projects directly and robustly to both the frontal cortex (specifically, the whisker region of motor cortex (wMC)) and the dorsolateral striatum (DLS) (Mao, 2011). Previous studies of wMC and DLS during whisker-based operant tasks have demonstrated their likely involvement in task performance. From neural recordings during whisker stimulus detection tasks, we have previously identified wMC as demonstrating sensory-motor gating (Zagha et al., 2015), distractor stimulus attenuation (Aruljothi et al., 2020), and target stimulus amplification with early and sustained choice probability (Zareian et al., 2021). These findings from wMC are similar to findings from premotor cortices in non-human primate studies (de Lafuente \& Romo, 2006). Inactivation of motor cortices in rodents results in impaired motor choice and execution (Morandell \& Huber, 2017) and impaired response suppression (Ebbesen \& Brecht, 2017; Murakami et al., 2017; Zagha et al., 2015). However, other studies have found motor cortex dispensable for the performance of learned tasks (Hwang et al., 2021; Kawai et al., 2015). During performance in other whisker detection/discrimination tasks, DLS neurons have demonstrated strong choice probability (Sippy et al., 2015). Furthermore, stimulation of the DLS in well-trained mice evokes 
prepotent responses (Sippy et al., 2015), and lesioning of the DLS along with sensory cortex causes sustained deficits in task performance (Hong et al., 2018).

In our study, we compared the representations and functions of wMC and DLS in a Go/No-Go selective whisker detection task, in which mice learned to respond to target whisker deflections in one whisker field and ignore distractor whisker deflections in the opposite whisker field. In this task, we find that DLS and wMC differentially contribute to sensory selection and inhibition, despite having similar sensory and choice representations. 


\section{Method Details}

\section{Animals}

Experiments performed in this study were approved by the Institutional Animal Care and Use Committee of University of California, Riverside. Male and female mice of three strains were used for the experiments: wild type (C57BL/6J), Thy1-ChR2 and vGAT-ChR2 transgenic mice (all purchased from The Jackson Laboratory or bred in our own colony). Data from mice of each strain were combined, and the transgenic feature was not exploited in these studies. All mice were housed on a $12 \mathrm{~h}$ light/12h dark cycle. Food was always accessible to mice outside of the behavioral training sessions.

\section{Surgery}

All experiments were performed on head-fixed mice. To attach the headpost to the skull, the mice were first anesthetized with a mixture of ketamine $(100 \mathrm{mg} / \mathrm{kg})$, xylazine $(10 \mathrm{mg} / \mathrm{kg})$, and isoflurane (1-2\%) throughout the surgery. They were additionally administered meloxicam $(0.3$ $\mathrm{mg} / \mathrm{kg})$ and enrofloxacin $(5 \mathrm{mg} / \mathrm{kg})$ at the day of the surgery and for two days post-surgery. A 10 $\mathrm{mm} \times 10 \mathrm{~mm}$ part of scalp was resected. A stainless steel headpost with length of $3 \mathrm{~cm}$ and a weight of 1.5 grams with a central window of $8 \mathrm{~mm} \times 8 \mathrm{~mm}$, was attached to the skull using cyanoacrylate glue, super glue and in some cases dental cement. The $8 \mathrm{~mm} \times 8 \mathrm{~mm}$ exposed window was sealed with Kwik-sil. After the surgery, the mice recovered on a heating pad. Behavioral experiments were started a minimum of 3 days after recovery from surgery. At the day of recording or lesion, small craniotomies $(\sim 0.5 \mathrm{~mm})$ were made under isoflurane anesthesia to access the relevant cortical and subcortical regions (see below).

\section{Training}


We trained mice in a selective detection task, which we have characterized in previous studies (Aruljothi et al., 2020; Marrero et al., 2021; Zareian et al., 2021). The mice were head-fixed in a custom-made setup. Piezo-controlled paddles were placed symmetrically within bilateral whisker fields contacting multiple whiskers. Target and distractor whisker fields were assigned at the beginning of training (target as the right whisker field) and remained constant throughout. Sensory stimuli consisted of small, rapid deflections of either whisker field. The deflections ranged from 0.01-0.2 seconds (s) in duration with a velocity of $10 \mathrm{~mm} / \mathrm{s}$, always equal for target and distractor stimuli. For most of the training sessions, two different stimulus amplitudes were used, one (large) near the saturation of the mouse's psychometric range and the other (small) near the midpoint. Mice reported stimulus detection by licking a central lick port. A lockout of $0.2 \mathrm{~s}$ was imposed between stimulus onset and response window, and mice were punished with a timeout for responding during this delay. Following 5-9 s inter-trial intervals, mice received either target, distractor, or catch (no stimulus) trials. All licking outside the post-target response window were punished by time-out (resetting the inter-trial interval). Water rewards for responding during the post-target response window were delivered from the same lick port used to report stimulus detection. Mice were water restricted throughout the training period, with the goal of receiving all water during behavioral trainings. Supplemental water was given in the home cage if weights fell below $85 \%$ of initial weights. Behavioral training was implemented using custom MATLAB scripts and Arduino Uno boards to trigger task stimuli and report licking responses. For further details of the behavioral training and training stages, see (Aruljothi et al., 2020).

Mice were considered expert in the task once they achieved a target-distractor discrimination d-prime $>1$ for three consecutive days (d-prime=norminv (Hit rate)- norminv 
(False alarm rate), in which norminv is the inverse normal probability value). All recording and lesion experiments were performed in animals that had reached expert performance. For the electrophysiological recording sessions, performance values (discrimination d-prime) were as follows: target wMC: $2.7+/-0.6, \mathrm{n}=11$, distractor wMC: $2.5+/-0.6, \mathrm{n}=19$, target Str: $2.3+/-0.6$, $\mathrm{n}=10$, distractor Str: $2.3+/-0.7, \mathrm{n}=11$, target $\mathrm{wS} 1: 2.7+/-0.6, \mathrm{n}=17$, distractor $\mathrm{wS} 1: 2.9+/-0.6$ (mean +/- STD). Performance of the mice during muscimol inactivation are reported in the Results, since behavioral performance was the dependent variable under examination.

\section{Electrophysiological recordings}

20 mice were used for electrophysiological recording experiments. Recordings were conducted following at least 20 minutes after recovery from isoflurane anesthesia. Recovery was assessed based on normal mouse behavior within their home cage and high engagement during the first few minutes of the task. For wMC and DLS recordings, Neuronexus laminar probes with 16 sites and $100 \mu \mathrm{m}$ spacing were used (A1x16-5mm-100-177-OA16LP or A1x16-5mm-100-177-A16); for wS1 recordings, Neuronexus probes in clustered tetrode configuration were used (A1x16Poly2-5 mm-50s-177). For each recording, the probe was advanced slowly in the brain using hydraulic Narishige micromanipulators. Electrophysiology data were acquired using Neuralynx recording system and Cheetah viewer software. The data were acquired at $32 \mathrm{kHz}$, then subsequently filtered at 600 to $6000 \mathrm{~Hz}$ for spike analyses.

Craniotomies for wMC recordings were centered on $1 \mathrm{~mm}$ lateral, $1 \mathrm{~mm}$ anterior to bregma. These wMC coordinates were chosen to target the wS1 projection zone, as in our previous studies (Zareian et al., 2021). The probe was advanced until the last site was barely visible at the surface (16 sites spanning $1.5 \mathrm{~mm}$ within cortex and below). At these coordinates, the thickness of layer 1, 2/3 and 5A of wMC collectively span the superficial (dorsal) 500-600 
$\mu \mathrm{m}$ (Hooks et al., 2013). Therefore, we considered recording sites within this range as

'superficial wMC' and the more ventral recording sites as 'deep wMC.' DLS coordinates were as follows [from bregma]: $2.5 \mathrm{~mm}$ lateral, $0.34 \mathrm{~mm}$ posterior. The probe was advanced deep inside the DLS (distal tip 2300-2500 $\mu \mathrm{m}$ below the pial surface). The coordinates were initially chosen based on Allen Brain Institute Mouse Connectivity atlas, selecting the region within DLS receiving the highest density inputs from $\mathrm{wS} 1 . \mathrm{wS} 1$ coordinates were as follows [from bregma]: 2.3 to $4 \mathrm{~mm}$ lateral, 1 to $2.2 \mathrm{~mm}$ posterior. The probe recording sites spanned a $375 \mu \mathrm{m}$ range that was advanced to $500-1000 \mu \mathrm{m}$ below the pial surface. Manual whisker mapping was used to align the wS1 recording site to the whisker stimuli (Zareian et al., 2021).

\section{Muscimol Inactivation}

Muscimol injections of $2 \mathrm{mM}$ in normal saline were performed using the Nanoject III Programmable Nanoliter Injector from Drummond Scientific Company fitted with a borosilicate glass micropipette. The same surface coordinates were used for muscimol injections as described above for electrophysiological recordings. For wMC injections, $250 \mathrm{~nL}$ of muscimol was injected at a rate of $1-3 \mathrm{~nL} / \mathrm{s}$ at $1 \mathrm{~mm}$ deep (from the pial surface) and another $250 \mathrm{~nL}$ was administered at $0.5 \mathrm{~mm}$ deep, for a total of $500 \mathrm{~nL}$, to target both superficial and deep layers of wMC. For DLS injections, a single bolus of $250 \mathrm{~nL}$ of muscimol was injected at a rate of 1-3 $\mathrm{nL} / \mathrm{s}$ at $1.7 \mathrm{~mm}$ ventral.

For muscimol inactivation studies, expertly performing mice were assigned to alternating control performance days without muscimol exposure and experimental performance days with muscimol inactivation. Sites for muscimol inactivation were randomized for each mouse, such that the order of inactivation varied (both within and across mice). For daily behavioral testing (also see Supplemental Figure 5), mice were first placed in a classical conditioning version of 
our task (see (Aruljothi et al., 2020)) for 2 minutes to ensure licking responses were intact when presented with water reward. Then the full selective detection task was initiated (Supplemental Figure 6), and mice were permitted to perform the full task until unmotivated. After 1 hour of training, if mice collected fewer than 10 rewards, mice were placed back in the classical conditioning task for 15 minutes and licking to presented water rewards were monitored. If mice were still performing the full selective detection task after 1 hour, they were permitted to perform that task until unmotivated (as determined by time since previous reward greater than 10 minutes).

\section{Quantification and statistical analysis}

All the analyses were performed using SPSS custom MATLAB scripts and displayed using Corel Draw and Microsoft Excel. For all the statistical analyses, we used alpha $=0.05$ as significant threshold, unless otherwise stated.

\section{Multiunit activity (MUA) analyses}

Sessions were truncated to include a single engaged period (a continuous bout of at least 10 minutes of task performance with no gaps in responding (licking) greater than 60 seconds). For wMC and DLS sessions, MUA was identified as negative-going threshold crossings over $3 \times$ standard deviation of the band-pass filtered voltage fluctuations throughout that session. For wS1 sessions, MUA was obtained by averaging single unit activities (SUA). wS1 spiking was identified as negative-going threshold crossing over 18-40 mV. Single unit isolation for wS1 recordings was performed using SpikeSort3D and was further refined using manual inspection.

For wMC recordings, using linear multielectrode arrays with $100 \mu \mathrm{m}$ spacing, channels 1 to 7 (spanning the superficial $600 \mu \mathrm{m}$ ) were considered as superficial layers and channels 8 to 16 
as deep layers. For DLS data analyses, we first determined the location of the white matter, as the site within the middle 5-12 sites with the minimum average spiking. The recording sites below that were considered as putative DLS sites. These criteria were due to consideration of variation in positioning of the probe relative the exact location of the white matter and underlying DLS.

Spike counts were binned in $5 \mathrm{~ms}$ bins throughout each recording session and combined as needed for larger window analyses. We calculated sensory detection and choice probability values using signal detection theory (Zareian et al., 2021). Briefly, the sensory detection was calculated by considering the area under receiver operating characteristic (ROC) curve constructed from plotting cumulative distributions of spiking of a post-stimulus window against pre-stimulus baseline activity. For baseline activity, three consecutive epochs with the same size as the post-stimulus window were considered. For MUA latency analyses, as well as comparison between wMC and DLS latencies, $1 \mathrm{~s}$ before the stimulus was considered as baseline. Stimulus response latency was determined as the time to rise above 2 standard deviations above baseline. Two (out of 10) DLS recording sessions were excluded from the latency analyses, due to lack of post-stimulus responses above this threshold. Sensory encoding maps were generated from 100 ms sliding windows with $95 \%$ overlap (unless the maps were generated from non-overlapping windows which were explicitly mentioned). For average MUA and sensory detection values that were collapsed across channels, we considered a channel with maximum sensory encoding within the first $100 \mathrm{~ms}$ as the representative of that session. Only large stimuli were considered for these analyses.

Similarly, choice probability was calculated by the area under the ROC curve constructed from plotting cumulative distributions of spiking activity on hit trials against miss trials. The 
choice probability traces were calculated from 50 ms sliding windows with $90 \%$ overlap. For statistical comparisons, the session choice probability values were either compared to chance level $(50 \%)$ or to baseline choice probability of the sessions averaged over a $300 \mathrm{~ms}$ window before the stimulus onset. For collapsed choice probability values across channels, we considered a channel with maximum choice probability for each session. For choice probability analysis, sufficient hit and miss trials were assessed based on previously described criteria (Zareian et al., 2021). For all the statistical comparisons of recording experiments, either one-sample or twosample t-test were used.

\section{Muscimol inactivation analyses}

For the muscimol inactivation experiments, we obtained motivation duration, hit rate, false alarm rate, d-prime, criterion, and reaction time. Two distinct time windows were considered for the analysis of data: full 1-hour session, and 'engaged performance' time window. Engaged performance measures were based on the sessions in which mice were performing the task for at least 5 minutes with no lapse in licking greater than 1 minute. Data were averaged across all sessions and all mice for each set of analyses $(n=7$ total mice, see Table 1; discrepancies between the number of sessions used for those analyses were related to availability of number of trials in those sessions for the corresponding measure, with a minimum allowable of at least 1 trial). Motivation duration (Figure 5) was calculated as the sum of all times mice were active during the task, as defined by no lapse in licking greater than 1 minute. To compare effects of muscimol inactivation of different regions on performance measures, a one-way ANOVA was conducted between control and all experimental conditions (tDLS, dDLS, twMC, dwMC), and TukeyKramer post-hoc multiple comparison test was performed to determine significant differences between pairs of conditions. We also considered effects of regional versus lateralized 
bioRxiv preprint doi: https://doi.org/10.1101/2022.03.03.482906; this version posted March 4, 2022. The copyright holder for this preprint (which was not certified by peer review) is the author/funder, who has granted bioRxiv a license to display the preprint in perpetuity. It is made available under aCC-BY-NC 4.0 International license.

inactivation by running a 2-way ANOVA test; performance measures were grouped regionally

(main effect of wMC vs. DLS regions regardless of the hemisphere) and grouped by hemisphere

(main effect of target-aligned vs. distractor-aligned regions regardless of cortical or subcortical).

Interaction effects were also considered (regional $\times$ hemisphere).

\begin{tabular}{|l|r|r|r|r|r|r|r|}
\hline \multicolumn{1}{|c|}{$\begin{array}{c}\text { Inactivation } \\
\text { Experiment Type }\end{array}$} & \multirow{2}{*}{ (Fig. 5) } & \multirow{2}{*}{ (Fig. 6) } & \multirow{2}{*}{ (S.Fig. 3) } & \multicolumn{3}{|c|}{ Reaction Times (Supplemental Figure 4) } \\
\cline { 5 - 8 } Control & 72 & 72 & 72 & 72 & 72 & 72 & 70 \\
\hline tDLS & 10 & 10 & 7 & 7 & 6 & 6 & 6 \\
\hline twMC & 12 & 12 & 11 & 11 & 12 & 11 & 11 \\
\hline dDLS & 10 & 10 & 8 & 10 & 8 & 8 & 8 \\
\hline dwMC & 13 & 13 & 13 & 13 & 13 & 13 & 13 \\
\hline Total Sessions & 117 & 117 & 111 & 113 & 111 & 110 & 108 \\
\hline
\end{tabular}

Table 1. Session number (N) used for Muscimol Inactivation Experiment Analyses.

The number of sessions used to generate averaged data across sessions, across mice separated by type of inactivation experiment and corresponding datasets for each figure. Fig. 5: Motivation Duration; Fig.6: Target Large and Small Hit Rate. Distractor Large and Small False Alarm Rate, D Prime, and C; S. Fig. 3: Engaged Period - Target Large Hit Rate, Distractor Large False Alarm Rate; S. Fig. 4: Reaction Times (S. Fig. 4A: Target Large Stimulus Hits Reaction Time; S. Fig. 4C: Target Small Stimulus Hits Reaction Time; S. Fig. 4E: Distractor Large Stimulus False Alarms Reaction Time; S. Fig 4G: Distractor Small Stimulus False Alarms Reaction Time). 


\section{Results}

We trained mice in a head-fixed, whisker-based selective detection task (Aruljothi et al., 2020;

Marrero et al., 2021; Zareian et al., 2021). In this task, mice learned to respond (lick) to small, transient whisker deflections within one whisker field (target) and to ignore identical whisker deflections in the opposite whisker field (distractor) (Figure $1 \mathrm{~A}$ and F). Due to the lateralization of the somatosensory system, this task configuration establishes target-aligned and distractoraligned cortical and striatal fields that are symmetric across midline and contralateral to the deflected whiskers. In the task structure, we impose a $200 \mathrm{~ms}$ lockout between stimulus onset and response window, and mice learn to withhold responding across this delay. Mice are considered expert in this task once they achieve a separation (d-prime) between hit rate (response to target) and false alarm rate (response to distractor) greater than 1 for three consecutive days (for training details, see Methods and (Aruljothi et al., 2020)). Subsequently, electrophysiological recording and muscimol inactivation studies were conducted in expert mice while they were performing the selective detection task, as described below.

\section{Laminar characterization of sensory responses and distractor attenuation in bilateral whisker motor cortex (wMC)}

A neural correlate of distractor inhibition is the attenuation of distractor compared to target stimulus responses (Moran \& Desimone, 1985; Treisman, 1964). In a previous widefield $\mathrm{Ca}^{2+}$ imaging study of the same task, we localized within neocortex the site of robust distractor attenuation to wMC (Aruljothi et al., 2020). Here, we used the higher spatio-temporal affordances of laminar electrophysiological recordings to further localize target and distractor 
stimulus responses across cortical layers within wMC (Figure 1). Multiunit activity (MUA) signals appeared at short latency in superficial and deep layers, for both target stimuli in targetaligned wMC (Figure 1C, D) and for distractor stimuli in distractor-aligned wMC (Figure 1H, I). A notable difference, however, was the strong and sustained activation of deep layers in targetaligned wMC, compared to the modest and transient activation of deep layers in distractor aligned wMC (Figure 1D, E, I, J). Averaging across all recording sessions, target-aligned sensory encoding within the first $100 \mathrm{~ms}$ post-stimulus (which is $100 \mathrm{~ms}$ before the earliest reaction times) peaked at a neurometric d-prime (see Methods) of $1.29+/-0.13$ at $1.1 \mathrm{~mm}$ from the pial surface (Figure 1D and E), corresponding to deep layer 5B. Distractor-aligned sensory encoding peaked at a neurometric d-prime of $0.54+/-0.08$ at $0.9 \mathrm{~mm}$ from the pial surface (Figure 1I and J).

To quantify distractor attenuation, we separated each laminar recording into superficial $(<600 \mu \mathrm{m})$ and deep $(>600 \mu \mathrm{m})$ layers of wMC. For each set, we selected the site with maximum sensory encoding for further analyses. For example, Figure 2B shows the average MUA across all recording sessions for the strongest target encoding sites per recording session in deep layers of wMC. We then compared target-aligned encoding of target stimuli to distractor-aligned encoding of distractor stimuli, for superficial and deep layers of wMC. We found a significant reduction of distractor encoding in deep layers of wMC (deep layers of target-aligned wMC: dprime mean $=1.53+/-0.09, \mathrm{n}=11$ sessions, deep layers of distractor-aligned wMC, d-prime mean $=0.9+/-0.07, \mathrm{n}=19$ sessions, two-sample t-test $\mathrm{p}$-value $<0.001$; reflecting a reduction in distractor encoding of $41 \%$ ) (Figure $2 \mathrm{G}$, top). In contrast, we found highly similar target and distractor encoding in superficial layers of wMC (superficial layers of target-aligned wMC: dprime mean $=0.66+/-0.12, \mathrm{n}=11$ sessions, superficial layers of distractor-aligned wMC, d-prime 
mean $=0.64+/-0.09, \mathrm{n}=19$, two-sample t-test $\mathrm{p}$-value $=0.89$; reflecting a reduction in distractor encoding of 3\%). Thus, these findings further localize neocortical attenuation specifically to deep layers of wMC.

\section{Similar sensory responses and distractor attenuation between deep layers of wMC and dorsolateral striatum (DLS)}

We performed similar spiking, stimulus encoding, and attenuation analyses for the DLS (Figure 2D). In figure $2 \mathrm{E}$, we show the average MUA across all target-aligned DLS recordings sessions for the recording site with the strongest target stimulus encoding. We compared the time course of spiking activity between wMC and DLS, plotting average spike rates in non-overlapping temporal windows varying from $5 \mathrm{~ms}$ to $100 \mathrm{~ms}$ (Figure $2 \mathrm{C}$ and $\mathrm{F}$ ). wMC and DLS displayed remarkably similar temporal response profiles, both peaking at 40-45 ms post-stimulus onset. Additionally, target stimulus response latencies were similar between wMC and DLS (wMC latency $=25.45+/-3.78 \mathrm{~ms}, \mathrm{n}=11$, DLS latency=25.00+2.31 ms, $\mathrm{n}=8, \mathrm{p}=0.93$, two sample $\mathrm{t}$-test). Similar post-stimulus and pre-response activity profiles between wMC and DLS were also evident when trials were aligned based on their reaction time (Supplemental Figure 1).

Next, we quantified maximum target and distractor stimulus encoding in target-aligned and distractor-aligned DLS, respectively (Figure 2G, bottom). We observed marginally significant yet robust distractor attenuation across the first $100 \mathrm{~ms}$ post-stimulus (target DLS: dprime mean $=1.50+/-0.22, \mathrm{n}=10$ sessions, distractor DLS, d-prime mean $=0.95+/-0.16, \mathrm{n}=11$ sessions, two-sample t-test $\mathrm{p}$-value $=0.05$ ), with a highly similar reduction of distractor encoding compared to deep layers of wMC (DLS 37\%, deep layers of wMC 41\%). Overall, we find 
stimulus response amplitude, time-course, and distractor attenuation to be highly similar between deep layers of wMC and DLS.

Given similar distractor attenuation in wMC and DLS, we considered whether this was already present in a common upstream structure, the whisker region of primary somatosensory cortex (wS1). First, we compared the time course of target and distractor encoding in stimulusaligned wS1, wMC, and DLS. As shown in Figure 3, wS1, deep layers of wMC, and DLS all show significant divergence of target and distractor encoding within the first $100 \mathrm{~ms}$ poststimulus (whereas superficial layers of wMC does not). Importantly, the divergence in deep layers of wMC and DLS precede divergence within wS1 (earliest significant difference in target versus distractor stimulus encoding: wS1, 55 ms; deep layers of wMC, 40 ms; DLS, $35 \mathrm{~ms}$ ). We obtained similar findings when considering different binning windows (Supplemental Figure 2). Thus, distractor attenuation may be implemented within wMC and/or DLS, but it is not simply inherited from wS1.

\section{Similar early choice encoding in target-aligned deep layers of wMC and target-aligned DLS}

Spiking activity in target-aligned wMC and DLS both showed strong sensory and motor features, with prominent post-stimulus and pre-response temporal alignments (Supplemental Figure 1)

(Zareian et al., 2021). Therefore, we used choice probability analyses to gain insights into the temporal latencies of the sensory-motor transformations within each region. Choice probability is the quantification of a relationship between neuronal activity and behavioral outcome, independent of stimulus amplitude (Britten et al., 1996). Early high choice probability is more likely to reflect a decision-making process than late choice probability, which may reflect the 
consequence of a decision established elsewhere (Crapse \& Basso, 2015; Siegel et al., 2015).

Here, we use choice probability latency as a measure of target stimulus selection (Figure 4). In

Figure 4A, we plot the choice probability time course for target stimuli, as the separation of spiking activity on hit versus miss trials, in target-aligned and distractor-aligned wMC and DLS. Deep layers of target-aligned wMC and target-aligned DLS both show prominent early choice probability peaks within $100 \mathrm{~ms}$ post-stimulus (Figure 4A, C, G). The finding in wMC is consistent with our previous report of early choice probability in layer 5 of wMC (Zareian et al., 2021). Furthermore, these latencies in target-aligned wMC and DLS are earlier than our and other reports of late choice probability (>100 ms) in wS1 (Sachidhanandam et al., 2013; Zareian et al., 2021). Other regions analyzed here showed smaller early peaks (target-aligned superficial layers of $\mathrm{wMC}$ ) or more gradual rises in choice probability, peaking during the response window $>200$ ms post-stimulus onset (distractor-aligned deep and superficial layers of wMC and distractor-aligned DLS). Thus, both deep layers of wMC and DLS (target-aligned) may be contributing to the early selection of target stimuli and their transformation to motor responses.

We recently reported lower than chance (negative) choice probability before stimulus onset throughout neocortex, indicating that lower prestimulus activity is more likely to result in a response (versus no response) outcome (Marrero et al., 2021). Interestingly, here we observe below chance pre-stimulus choice probability in target-aligned and distractor-aligned DLS as well (Figure 4A, F, H). Consequently, above chance post-stimulus choice probability in DLS was observed only after normalizing for this reduced pre-stimulus baseline (Figure 4G and I). These pre-stimulus baseline differences can also be appreciated in plots of the average spike rates preceding hit and miss trials (Supplemental Figure 1C and 1F). 
In summary, we find remarkable similarities in sensory-related and choice-related signals in deep layers of wMC and DLS. In particular, robust distractor attenuation and target choice probability in both wMC and DLS suggests that activities in either or both regions may be driving task performance.

\section{Task performance requires target-aligned DLS}

Next, we performed muscimol (GABAA receptor agonist) inactivation studies to determine the essential functional contributions of wMC and DLS to task performance. Muscimol provides stable inactivation of the exposed region during behavioral testing, enabling assessment of its essential functions that cannot be compensated for on the order of minutes to hours (with full recovery of neural activity within 6 hours, (Arikan et al., 2002)). In mice that had achieved expert performance, we alternated muscimol injected and control (non-injected) training sessions, randomizing muscimol inactivation among the four regions (target-aligned DLS (tDLS), target-aligned wMC (twMC), distractor-aligned DLS (dDLS), distractor-aligned wMC (dwMC); injections into wMC were directed to both superficial and deep layers). Analyses consisted of one-way ANOVA, to compare each condition separately, and two-way ANOVA, to compare brain regions (wMC versus DLS) and hemisphere alignment (target-aligned versus distractor-aligned) main effects and interactions (brain regions $\times$ hemisphere alignment).

The first outcome measure we assessed was motivation duration (Figure 5), quantified as the total time of engagement in the selective detection task (see Methods for details). We found that inactivation of target-aligned DLS significantly and drastically reduced motivation duration, by $72 \%$ compared to control levels (control: mean duration 5460 +/- 270 seconds, 
target-aligned DLS: mean duration 1510 +/- 470 seconds, Tukey-Kramer post-hoc comparison, $\mathrm{p}<0.001$ ) (Figure 5A). A 2-way ANOVA analysis determined significant main effects of neural structure $(\mathrm{p}=0.001)$ and alignment $(\mathrm{p}=0.033)$, with DLS and target-alignment reducing motivation duration (Figure 5B).

To better understand the contributions of each region to task performance, we additionally assessed effects of inactivation on response rates, behavioral d-prime, and criterion (standardized to 1 hour in task, see Methods for details) (Figure 6). For target and distractor trials, we separately analyzed response rates for large amplitude and small amplitude stimuli (Figure 6A-H). Target-aligned DLS inactivation robustly reduced response rates to target stimuli, compared to control levels (large hit rate: $65 \%$ reduction, control: $0.71+/-0.03$, tDLS: $0.25+/-$ $.11, \mathrm{p}<0.001$, Figure 6A; small hit rate, 63\% reduction, control: 0.52 +/- 0.03, tDLS: 0.19 +/$0.09, \mathrm{p}=0.012$, Figure 6C). Notably, reductions in large hit rates were also observed when only considering continuously engaged task epochs (Supplemental Figure 3A). Furthermore, targetaligned DLS inactivation resulted in non-significant trends in reduced response rates to distractor stimuli (large false alarm rate: 59\% reduction, control: $0.16+/-0.01$, tDLS: $0.06+/-0.02$, $\mathrm{p}=0.260$, Figure $6 \mathrm{E}$; small false alarm rate, $63 \%$ reduction, control: $0.16+/-0.14$, tDLS: 0.06 +/0.02, $\mathrm{p}=0.088$, Figure 6G). Target-aligned DLS inactivation reduced behavioral d-prime (control: $1.91+/-.11$, tDLS: $0.56+/-.31, \mathrm{p}=0.001)$ (Figure 6I), and, perhaps most characteristically, drastically increased criterion (control, $0.13+/-0.08$, target-aligned DLS inactivation, $1.43+/-$ $0.30, \mathrm{p}<0.001)$

As described above, inactivation of target-aligned DLS dramatically reduced the tendency to respond to task-relevant stimuli. However, from these data we cannot yet determine if target-aligned DLS is playing a specific role in linking task-relevant stimuli to the prepotent 
response, or whether it has a more global role in motivation or response initiation/execution. The former would predict intact responding if triggered by a different pathway, whereas the latter would predict a comprehensive response deficit. To distinguish between these possibilities, we tested these mice in a classical conditioning task in which an auditory cue the mice had previously associated with reward (opening of a solenoid) was presented along with a fluid reward (Supplemental Figure 5). In 8 out of 10 target-aligned DLS inactivation sessions, mice did respond at high rates $(>50 \%)$ to the auditory cue (Supplemental Figure 7$)$, arguing against a global deficit in motivation or movement. These data suggest that target-aligned DLS is functioning as a critical node in the selection of task-relevant stimuli for linking to the prepotent response.

\section{Distractor-aligned wMC makes essential contributions to distractor suppression}

In stark contrast to the effects of target-aligned DLS inactivation, inactivation of distractoraligned wMC increased response rates. This was most dramatic for large distractor stimuli, increasing false alarm rates by $84 \%$ compared to control sessions (control: $0.16+/-0.01$, dwMC inactivation: $0.29+/-0.05, \mathrm{p}=0.013$ ) (Figure 6E). This effect was also present when only considering continuously engaged task epochs (large false alarm rate: 63\% increase, control: 0.15+/-0.01, dwMC: 0.25+/-0.01, p=0.013) (Supplemental Figure 3B). While we observed trends towards increased response rates for all other stimuli (Figure 6A, C, G), these distributions compared to controls were not statistically significant. For multiple measures, effects of targetaligned DLS inactivation versus distractor-aligned wMC inactivation were significantly different from each other and opposite in direction compared to control sessions. This includes large target hit rate (tDLS: $0.25+/-.11$, dwMC: $0.82+/-0.07, \mathrm{p}=<0.001$ ) (Figure 6A), large distractor false 
alarm rate (tDLS: 0.64+/-0.02, dwMC: 0.29+/-0.05, p=0.001) (Figure 6E), criterion (tDLS: 1.43

+/- 0.30, dwMC: $-0.33+/-0.20, \mathrm{p}=<0.001)$ (Figure 6K), and large target reaction times (tDLS:

0.533+/-0.098 s, dwMC: 0.335+/-0.025 s, p=0.018) (Supplemental Figure 4A). These findings

highlight the distinct functional contributions of these two regions on task performance.

Essential contributions of target-aligned wMC and distractor-aligned DLS are less clear. For both inactivation datasets, there were non-significant trends (compared to control sessions) for reduced motivation duration and reduced large and small target hit rates (Figure 6A and C). Such findings suggest possible contributions to sensory selection. However, target-aligned wMC inactivation resulted in a non-significant trend towards increased large distractor false alarm rates (Figure 6E), and thus may also contribute to distractor inhibition. 


\section{Discussion}

\section{Distributed functions of frontal cortex and dorsolateral striatum}

In this study we characterize selection and inhibition within key regions of the frontal cortex and dorsolateral striatum, collectively known as the frontostriatal network (Frank, 2011; Terra et al., 2020). In deep layers of wMC and the DLS we find highly similar sensory (Figures 2 and 3), choice (Figure 4) and motor (Supplemental Figure 1) representations. Nonetheless, our inactivation experiments provide evidence for distinct functions of wMC and DLS in selection (Figure 5 and 6) and inhibition (Figure 6) of sensory responses. Collectively, our data supports that DLS acts as a crucial bottleneck in linking task-relevant stimuli to prepotent responses, while wMC contributes to distractor stimulus inhibition (Figure 7).

Our findings contrast with traditional views of cortical and striatal function in important ways. First, motor cortices are generally understood as primarily triggering motor actions (Georgopoulos et al., 1982; Kalaska et al., 1989; Schwartz et al., 1988). However, this study is consistent with a growing literature, primarily in rodents, suggesting essential roles of motor cortices in suppressing prepotent responses (Ebbesen \& Brecht, 2017; Murakami et al., 2017; Zagha et al., 2015). While response inhibition is not commonly associated with motor cortices, it is a well-established function of frontal cortex in general across a range of species (Aron et al., 2003; Aron et al., 2014; Brainin et al., 2008; Chen et al., 2010; Ebbesen \& Brecht, 2017; Konorski, 1972; Nachev et al., 2008; Stuphorn et al., 2010; Swick et al., 2008). And yet, we do not believe that response inhibition is the sole function of motor cortex in rodents. Ample evidence, across species, supports motor cortex involvement in the learning and execution of skilled movements (Gandolfo et al., 2000; Hikosaka et al., 1995; Hwang et al., 2021; Kawai et 
al., 2015; Lemke et al., 2021; Muellbacher et al., 2002; Nakamura et al., 1998, 1999; Puzerey et al., 2018). A more general framework of motor cortex is its involvement in 'motor control', which includes both motor activation and suppression as needed for performing a goal-directed task.

The role of striatum in linking task-relevant stimuli to a prepotent response does match traditional views of striatal control of action selection (Gerfen et al., 1990; Grillner et al., 2005; Mink, 1996; Redgrave et al., 1999). However, in these frameworks, striatal selection is propagated back to motor cortex, which ultimately triggers response execution (Houk et al., 2007; Nakano et al., 2000). If striatal selection did require execution by motor cortex, then inactivation of wMC would have caused equal or greater disruption of sensory selection than inactivation of DLS. In contrast, our data suggest that striatum can directly trigger response execution, without the involvement of motor cortex (as illustrated in Figure 7). One alternative possibility is that different regions of motor cortex can compensate for the inactivation of wMC. And yet, there is growing appreciation of direct projections from the basal ganglia to subcortical motor structures, including superior colliculus and brainstem motor centers (Lee \& Sabatini, 2021; Saitoh et al., 2003; Takakusaki et al., 2003; Takakusaki et al., 2011). We propose that, at least in the rodent, striatal selection does not require motor cortex for response execution.

Our circuit model (Figure 7) does contrast with a recent study comparing the circuit-level and behavioral impacts of wS1 and wMC onto DLS. That study concluded that wS1 suppresses response execution by inhibiting DLS through the activation of local interneurons, whereas wMC drives response execution by activating DLS projection neurons (Lee et al., 2019). Future studies are needed to determine whether these apparent discrepancies are due to differences in 
the methods used, or due to functional adaptations of the cortico-striatal system in the context of passive whisker detection (this study) versus active sensory discrimination (Lee et al., 2019).

\section{Mechanisms of distractor inhibition and target selection}

From the perspective of signal routing (Zagha, 2020), distractor inhibition can simply be understood as the failure to forward propagate a sensory response, such that it does not trigger a motor action. We find robust distractor response attenuation in both DLS and deep layers of wMC. Interestingly, we did not find robust distractor attenuation in superficial layers of wMC. This laminar specialization in wMC has two important implications. First, this finding of distractor attenuation in wMC would have been missed if using somatic imaging approaches, which are often limited to sampling from superficial layers. Second, wS1 axons project strongly to wMC neurons in superficial layers, and only weakly to wMC neurons in deep layers (Mao et al., 2011). The differential (target vs distractor) activation of deep layer wMC neurons suggests that sensory amplification/attenuation is implemented by intracortical processing within wMC. The contributions of top-down modulation and bottom-up plasticity, to wMC (Chen et al., 2013; Chen et al., 2015) and DLS (Xiong et al., 2015), in mediating amplification/attenuation is a focus of ongoing investigation.

While regulated sensory propagation may be one aspect of distractor inhibition, a second aspect appears to be response triggering. Inactivation of target-aligned DLS compared to distractor-aligned DLS tended to reduce responses to both target and distractor stimuli (not statistically significant, but consistent trends, Figures 5A, C, E, G). Thus, we speculate that through learning, response triggering for target and distractor stimuli becomes conditioned on the activation of target-aligned DLS. Understanding the neural mechanisms underlying this conditioned response triggering, and its possible dependence on dopamine neuromodulation 
(Gerfen et al., 1990; Gerfen \& Surmeier, 2011; Kravitz et al., 2010; Surmeier et al., 1996), is an important topic of future research.

It was recently proposed that baseline (prestimulus) activity within the striatum may regulate task engagement, with higher prestimulus activity priming motor execution by bringing the network closer to response threshold (Steinmetz et al., 2019). This framework would have predicted positive prestimulus choice probability for target-aligned DLS. In contrast, we find that bilateral DLS, like the rest of dorsal neocortex (Marrero et al., 2021), displays negative prestimulus choice probability in response to target stimuli (Figure 4A, F and Supplemental Figure 1F). This difference may be due to sensory modality or task design. Nonetheless, our data suggest that for both cortical and subcortical structures in the whisker system, noise reduction enhances stimulus detection.

\section{Dissociation between representation and function}

More generally, our findings bring up important considerations about interpreting representational and causal data. Multiple studies have shown dissociations between representation and function, such that regions with strong representation have only minor impacts on behavior when inactivated (e.g., Hong et al., 2018; Katz et al., 2016; Schiller et al., 1980; Zatka-Haas et al., 2021). This finding may be partially explained by the redundancy of neural representations in simple decision-making tasks (de Lafuente \& Romo, 2006; Gold \& Shadlen, 2007; Hanks et al., 2015; Steinmetz et al., 2019). We also identify redundant neural representation in this study. The early positive post-stimulus choice probability in deep layers of wMC described here and previously (Zagha et al., 2015; Zareian et al., 2021) appears to be redundant and dispensable for response execution, due to the parallel processing of these signals in the DLS. 
And yet, dissociation between representation and function is not entirely accounted for by redundancy. A core finding of this study is that distractor-aligned wMC contributes to response inhibition. Response inhibition may be mediated by post-stimulus activation (Aron et al., 2003; Aron et al., 2014; Eagle et al., 2007), presumably to suppress motor triggering (Hanes et al., 1998; Logan \& Cowan, 1984; Logan et al., 2014). This mechanism would be associated with a negative post-stimulus choice probability, in which activation predicts response suppression. In contrast, our distractor-aligned wMC recordings demonstrate a long-latency positive poststimulus choice probability in response to target trials (which likely reflects movement-related signaling during the response, (Zagha et al., 2022)) and chance level choice probability in response to distractor stimuli (data not shown). This discrepancy between representation and function may suggest an alternative mechanism of response inhibition, in which the baseline (pre-stimulus) activity tonically and pro-actively suppresses response triggering. If this is indeed the case, then this discrepancy between representation and function may itself reveal a specific neural mechanism. 


\section{References}

Alexander, G. E., \& Crutcher, M. D. (1990). Functional architecture of basal ganglia circuits: neural substrates of parallel processing. Trends in neurosciences, 13(7), 266-271.

Arikan, R., Blake, N. M., Erinjeri, J. P., Woolsey, T. A., Giraud, L., \& Highstein, S. M. (2002). A method to measure the effective spread of focally injected muscimol into the central nervous system with electrophysiology and light microscopy. Journal of neuroscience methods, 118(1), 51-57.

Aron, A. R., Fletcher, P. C., Bullmore, E. T., Sahakian, B. J., \& Robbins, T. W. (2003). Stopsignal inhibition disrupted by damage to right inferior frontal gyrus in humans. Nature Neuroscience, 6(2), 115-116. https://doi.org/10.1038/nn1003

Aron, A. R., Robbins, T. W., \& Poldrack, R. A. (2014). Inhibition and the right inferior frontal cortex: one decade on. Trends in cognitive sciences, 18(4), 177-185.

Aruljothi, K., Marrero, K., Zhang, Z., Zareian, B., \& Zagha, E. (2020). Functional localization of an attenuating filter within cortex for a selective detection task in mice. Journal of Neuroscience, 40(28), 5443-5454.

Avanzino, L., Fiorio, M., \& Conte, A. (2018). Actual and illusory perception in Parkinson's disease and dystonia: a narrative review. Frontiers in neurology, 9, 584.

Baranek, G. T., Watson, L. R., Boyd, B. A., Poe, M. D., David, F. J., \& McGuire, L. (2013). Hyporesponsiveness to social and nonsocial sensory stimuli in children with autism, children with developmental delays, and typically developing children. Development and psychopathology, 25(2), 307-320.

Bergstrom, H. C., Lieberman, A. G., Graybeal, C., Lipkin, A. M., \& Holmes, A. (2020). Dorsolateral striatum engagement during reversal learning. Learning \& Memory, 27(10), 418-422.

Bergstrom, H. C., Lipkin, A. M., Lieberman, A. G., Pinard, C. R., Gunduz-Cinar, O., Brockway, E. T., Taylor, W. W., Nonaka, M., Bukalo, O., \& Wills, T. A. (2018). Dorsolateral striatum engagement interferes with early discrimination learning. Cell reports, 23(8), 2264-2272.

Brainin, M., Seiser, A., \& Matz, K. (2008). The mirror world of motor inhibition: the alien hand syndrome in chronic stroke. J Neurol Neurosurg Psychiatry, 79(3), 246-252. https://doi.org/10.1136/jnnp.2007.116046

Britten, K. H., Newsome, W. T., Shadlen, M. N., Celebrini, S., \& Movshon, J. A. (1996). A relationship between behavioral choice and the visual responses of neurons in macaque MT. Visual neuroscience, 13(1), 87-100.

Chen, J. L., Carta, S., Soldado-Magraner, J., Schneider, B. L., \& Helmchen, F. (2013). Behaviour-dependent recruitment of long-range projection neurons in somatosensory cortex. Nature, 499(7458), 336-340. https://doi.org/10.1038/nature12236

Chen, J. L., Margolis, D. J., Stankov, A., Sumanovski, L. T., Schneider, B. L., \& Helmchen, F. (2015). Pathway-specific reorganization of projection neurons in somatosensory cortex during learning. Nat Neurosci, 18(8), 1101-1108. https://doi.org/10.1038/nn.4046

Chen, X., Scangos, K. W., \& Stuphorn, V. (2010). Supplementary motor area exerts proactive and reactive control of arm movements. J Neurosci, 30(44), 14657-14675. https://doi.org/10.1523/jneurosci.2669-10.2010 
Cools, R., Clark, L., \& Robbins, T. W. (2004). Differential responses in human striatum and prefrontal cortex to changes in object and rule relevance. Journal of Neuroscience, 24(5), 1129-1135.

Crapse, T. B., \& Basso, M. A. (2015). Insights into decision making using choice probability. Journal of neurophysiology, 114(6), 3039-3049.

de Lafuente, V., \& Romo, R. (2006). Neural correlate of subjective sensory experience gradually builds up across cortical areas. Proceedings of the National Academy of Sciences, 103(39), 14266-14271.

Eagle, D. M., Baunez, C., Hutcheson, D. M., Lehmann, O., Shah, A. P., \& Robbins, T. W. (2007). Stop-Signal Reaction-Time Task Performance: Role of Prefrontal Cortex and Subthalamic Nucleus. Cerebral Cortex, 18(1), 178-188. https://doi.org/10.1093/cercor/bhm044

Ebbesen, C. L., \& Brecht, M. (2017). Motor cortex - to act or not to act? Nat Rev Neurosci, 18(11), 694-705. https://doi.org/10.1038/nrn.2017.119

Ford, J. M., Roach, B. J., Jorgensen, K. W., Turner, J. A., Brown, G. G., Notestine, R., BischoffGrethe, A., Greve, D., Wible, C., \& Lauriello, J. (2009). Tuning in to the voices: a multisite FMRI study of auditory hallucinations. Schizophrenia bulletin, 35(1), 58-66.

Frank, M. J. (2011). Computational models of motivated action selection in corticostriatal circuits. Curr Opin Neurobiol, 21(3), 381-386. https://doi.org/10.1016/j.conb.2011.02.013

Gandolfo, F., Li, C.-S. R., Benda, B. J., Schioppa, C. P., \& Bizzi, E. (2000). Cortical correlates of learning in monkeys adapting to a new dynamical environment. Proceedings of the National Academy of Sciences, 97(5), 2259-2263. https://doi.org/doi:10.1073/pnas.040567097

Georgopoulos, A., Kalaska, J., Caminiti, R., \& Massey, J. (1982). On the relations between the direction of two-dimensional arm movements and cell discharge in primate motor cortex. The Journal of Neuroscience, 2(11), 1527-1537. https://doi.org/10.1523/jneurosci.02-1101527.1982

Gerfen, C. R., Engber, T. M., Mahan, L. C., Susel, Z., Chase, T. N., Monsma, F. J., Jr., \& Sibley, D. R. (1990). D1 and D2 dopamine receptor-regulated gene expression of striatonigral and striatopallidal neurons. Science, 250(4986), 1429-1432. https://doi.org/10.1126/science. 2147780

Gerfen, C. R., \& Surmeier, D. J. (2011). Modulation of Striatal Projection Systems by Dopamine. Annual Review of Neuroscience, 34(1), 441-466. https://doi.org/10.1146/annurev-neuro-061010-113641

Gold, J. I., \& Shadlen, M. N. (2007). The neural basis of decision making. Annu Rev Neurosci, 30, 535-574. https://doi.org/10.1146/annurev.neuro.29.051605.113038

Graybiel, A. M., Aosaki, T., Flaherty, A. W., \& Kimura, M. (1994). The basal ganglia and adaptive motor control. Science, 265(5180), 1826-1831.

Grillner, S., Hellgren, J., Menard, A., Saitoh, K., \& Wikström, M. A. (2005). Mechanisms for selection of basic motor programs-roles for the striatum and pallidum. Trends in neurosciences, 28(7), 364-370.

Hanes, D. P., Patterson, W. F., 2nd, \& Schall, J. D. (1998). Role of frontal eye fields in countermanding saccades: visual, movement, and fixation activity. J Neurophysiol, 79(2), 817-834. https://doi.org/10.1152/jn.1998.79.2.817 
Hanes, D. P., \& Schall, J. D. (1996). Neural control of voluntary movement initiation. Science, 274(5286), 427-430.

Hanks, T. D., Kopec, C. D., Brunton, B. W., Duan, C. A., Erlich, J. C., \& Brody, C. D. (2015). Distinct relationships of parietal and prefrontal cortices to evidence accumulation. Nature, 520(7546), 220-223. https://doi.org/10.1038/nature14066

Hikosaka, O., Rand, M. K., Miyachi, S., \& Miyashita, K. (1995). Learning of sequential movements in the monkey: process of learning and retention of memory. J Neurophysiol, 74(4), 1652-1661. https://doi.org/10.1152/jn.1995.74.4.1652

Hong, Y. K., Lacefield, C. O., Rodgers, C. C., \& Bruno, R. M. (2018). Sensation, movement and learning in the absence of barrel cortex. In Nature (Vol. 561, pp. 542-546): Nature Publishing Group.

Hooks, B. M., Mao, T., Gutnisky, D. A., Yamawaki, N., Svoboda, K., \& Shepherd, G. M. (2013). Organization of cortical and thalamic input to pyramidal neurons in mouse motor cortex. Journal of Neuroscience, 33(2), 748-760.

Houk, J. C., Bastianen, C., Fansler, D., Fishbach, A., Fraser, D., Reber, P. J., Roy, S. A., \& Simo, L. S. (2007). Action selection and refinement in subcortical loops through basal ganglia and cerebellum. Philos Trans R Soc Lond B Biol Sci, 362(1485), 1573-1583. https://doi.org/10.1098/rstb.2007.2063

Hwang, E. J., Dahlen, J. E., Mukundan, M., \& Komiyama, T. (2021). Disengagement of motor cortex during long-term learning tracks the performance level of learned movements. Journal of Neuroscience, 41(33), 7029-7047.

Jin, X., \& Costa, R. M. (2010). Start/stop signals emerge in nigrostriatal circuits during sequence learning. Nature, 466(7305), 457-462.

Kalaska, J., Cohen, D., Hyde, M., \& Prud'homme, M. (1989). A comparison of movement direction-related versus load direction- related activity in primate motor cortex, using a two-dimensional reaching task. The Journal of Neuroscience, 9(6), 2080-2102. https://doi.org/10.1523/jneurosci.09-06-02080.1989

Katz, L. N., Yates, J. L., Pillow, J. W., \& Huk, A. C. (2016). Dissociated functional significance of decision-related activity in the primate dorsal stream. Nature, 535(7611), 285-288. https://doi.org/10.1038/nature18617

Kawai, R., Markman, T., Poddar, R., Ko, R., Fantana, A. L., Dhawale, A. K., Kampff, A. R., \& Ölveczky, B. P. (2015). Motor cortex is required for learning but not for executing a motor skill. Neuron, 86(3), 800-812.

Konorski, J. (1972). Some hypotheses concerning the functional organization of prefrontal cortex. Acta Neurobiol Exp (Wars), 32(2), 595-613.

Kravitz, A. V., Freeze, B. S., Parker, P. R., Kay, K., Thwin, M. T., Deisseroth, K., \& Kreitzer, A. C. (2010). Regulation of parkinsonian motor behaviours by optogenetic control of basal ganglia circuitry. Nature, 466(7306), 622-626. https://doi.org/10.1038/nature09159

Lee, C. R., Yonk, A. J., Wiskerke, J., Paradiso, K. G., Tepper, J. M., \& Margolis, D. J. (2019). Opposing influence of sensory and motor cortical input on striatal circuitry and choice behavior. Current Biology, 29(8), 1313-1323. e1315.

Lee, J., \& Sabatini, B. L. (2021). Striatal indirect pathway mediates exploration via collicular competition. Nature, 599(7886), 645-649. https://doi.org/10.1038/s41586-021-04055-4

Lemke, S. M., Ramanathan, D. S., Darevksy, D., Egert, D., Berke, J. D., \& Ganguly, K. (2021). Coupling between motor cortex and striatum increases during sleep over long-term skill learning. elife, 10, e64303. 
Logan, G. D., \& Cowan, W. B. (1984). On the ability to inhibit thought and action: A theory of an act of control. Psychological Review, 91(3), 295-327. https://doi.org/10.1037/0033295X.91.3.295

Logan, G. D., Van Zandt, T., Verbruggen, F., \& Wagenmakers, E. J. (2014). On the ability to inhibit thought and action: general and special theories of an act of control. Psychol Rev, 121(1), 66-95. https://doi.org/10.1037/a0035230

Mao, T., Kusefoglu, D., Hooks, Bryan M., Huber, D., Petreanu, L., \& Svoboda, K. (2011). LongRange Neuronal Circuits Underlying the Interaction between Sensory and Motor Cortex. Neuron, 72(1), 111-123. https://doi.org/https://doi.org/10.1016/j.neuron.2011.07.029

Marrero, K., Aruljothi, K., Zareian, B., Gao, C., Zhang, Z., \& Zagha, E. (2021). Global, LowAmplitude Cortical State Predicts Response Outcomes in a Selective Detection Task in Mice. Cerebral Cortex. https://doi.org/10.1093/cercor/bhab339

Mink, J. W. (1996). The basal ganglia: focused selection and inhibition of competing motor programs. Prog Neurobiol, 50(4), 381-425. https://doi.org/10.1016/s03010082(96)00042-1

Moran, J., \& Desimone, R. (1985). Selective attention gates visual processing in the extrastriate cortex. Science, 229(4715), 782-784.

Morandell, K., \& Huber, D. (2017). The role of forelimb motor cortex areas in goal directed action in mice. Scientific reports, 7(1), 1-14.

Muellbacher, W., Ziemann, U., Wissel, J., Dang, N., Kofler, M., Facchini, S., Boroojerdi, B., Poewe, W., \& Hallett, M. (2002). Early consolidation in human primary motor cortex. Nature, 415(6872), 640-644. https://doi.org/10.1038/nature712

Murakami, M., Shteingart, H., Loewenstein, Y., \& Mainen, Z. F. (2017). Distinct sources of deterministic and stochastic components of action timing decisions in rodent frontal cortex. Neuron, 94(4), 908-919. e907.

Nachev, P., Kennard, C., \& Husain, M. (2008). Functional role of the supplementary and presupplementary motor areas. Nature Reviews Neuroscience, 9(11), 856-869. https://doi.org/10.1038/nrn2478

Nakamura, K., Sakai, K., \& Hikosaka, O. (1998). Neuronal activity in medial frontal cortex during learning of sequential procedures. J Neurophysiol, 80(5), 2671-2687. https://doi.org/10.1152/jn.1998.80.5.2671

Nakamura, K., Sakai, K., \& Hikosaka, O. (1999). Effects of local inactivation of monkey medial frontal cortex in learning of sequential procedures. J Neurophysiol, 82(2), 1063-1068. https://doi.org/10.1152/jn.1999.82.2.1063

Nakano, K., Kayahara, T., Tsutsumi, T., \& Ushiro, H. (2000). Neural circuits and functional organization of the striatum. J Neurol, 247 Suppl 5, V1-15. https://doi.org/10.1007/pl00007778

Owen, A. M., Doyon, J., Petrides, M., \& Evans, A. C. (1996). Planning and spatial working memory: a positron emission tomography study in humans. European journal of neuroscience, 8(2), 353-364.

Partiot, A., Verin, M., Pillon, B., Teixeira-Ferreira, C., Agid, Y., \& Dubois, B. (1996). Delayed response tasks in basal ganglia lesions in man: further evidence for a striato-frontal cooperation in behavioural adaptation. Neuropsychologia, 34(7), 709-721.

Pessoa, L. (2014). Understanding brain networks and brain organization. Physics of life reviews, $11(3), 400-435$. 
Peters, A. J., Fabre, J. M., Steinmetz, N. A., Harris, K. D., \& Carandini, M. (2021). Striatal activity topographically reflects cortical activity. Nature, 591(7850), 420-425.

Puzerey, P. A., Maher, K., Prasad, N., \& Goldberg, J. H. (2018). Vocal learning in songbirds requires cholinergic signaling in a motor cortex-like nucleus. Journal of neurophysiology, 120(4), 1796-1806. https://doi.org/10.1152/jn.00078.2018

Redgrave, P., Prescott, T. J., \& Gurney, K. (1999). The basal ganglia: a vertebrate solution to the selection problem? Neuroscience, 89(4), 1009-1023.

Sachidhanandam, S., Sreenivasan, V., Kyriakatos, A., Kremer, Y., \& Petersen, C. C. (2013). Membrane potential correlates of sensory perception in mouse barrel cortex. Nature neuroscience, 16(11), 1671-1677.

Saitoh, K., Hattori, S., Song, W. J., Isa, T., \& Takakusaki, K. (2003). Nigral GABAergic inhibition upon cholinergic neurons in the rat pedunculopontine tegmental nucleus. Eur $J$ Neurosci, 18(4), 879-886. https://doi.org/10.1046/j.1460-9568.2003.02825.x

Salinas, E., \& Romo, R. (1998). Conversion of sensory signals into motor commands in primary motor cortex. Journal of Neuroscience, 18(1), 499-511.

Schiller, P. H., True, S. D., \& Conway, J. L. (1980). Deficits in eye movements following frontal eye-field and superior colliculus ablations. Journal of Neurophysiology, 44(6), 11751189. https://doi.org/10.1152/jn.1980.44.6.1175

Schwartz, A., Kettner, R., \& Georgopoulos, A. (1988). Primate motor cortex and free arm movements to visual targets in three- dimensional space. I. Relations between single cell discharge and direction of movement. The Journal of Neuroscience, 8(8), 2913-2927. https://doi.org/10.1523/jneurosci.08-08-02913.1988

Siegel, M., Buschman, T. J., \& Miller, E. K. (2015). Cortical information flow during flexible sensorimotor decisions. Science, 348(6241), 1352-1355.

Sippy, T., Lapray, D., Crochet, S., \& Petersen, C. C. (2015). Cell-type-specific sensorimotor processing in striatal projection neurons during goal-directed behavior. Neuron, $88(2)$, 298-305.

Steinmetz, N. A., Zatka-Haas, P., Carandini, M., \& Harris, K. D. (2019). Distributed coding of choice, action and engagement across the mouse brain. Nature, 576(7786), 266-273. https://doi.org/10.1038/s41586-019-1787-X

Stephenson-Jones, M., Samuelsson, E., Ericsson, J., Robertson, B., \& Grillner, S. (2011). Evolutionary conservation of the basal ganglia as a common vertebrate mechanism for action selection. Current Biology, 21(13), 1081-1091.

Stuphorn, V., Brown, J. W., \& Schall, J. D. (2010). Role of supplementary eye field in saccade initiation: executive, not direct, control. J Neurophysiol, 103(2), 801-816. https://doi.org/10.1152/jn.00221.2009

Surmeier, D. J., Song, W. J., \& Yan, Z. (1996). Coordinated expression of dopamine receptors in neostriatal medium spiny neurons. J Neurosci, 16(20), 6579-6591. https://doi.org/10.1523/jneurosci.16-20-06579.1996

Swick, D., Ashley, V., \& Turken, A. U. (2008). Left inferior frontal gyrus is critical for response inhibition. BMC Neurosci, 9, 102. https://doi.org/10.1186/1471-2202-9-102

Takakusaki, K., Habaguchi, T., Ohtinata-Sugimoto, J., Saitoh, K., \& Sakamoto, T. (2003). Basal ganglia efferents to the brainstem centers controlling postural muscle tone and locomotion: a new concept for understanding motor disorders in basal ganglia dysfunction. Neuroscience, 119(1), 293-308. https://doi.org/10.1016/s03064522(03)00095-2 
Takakusaki, K., Obara, K., Nozu, T., \& Okumura, T. (2011). Modulatory effects of the GABAergic basal ganglia neurons on the PPN and the muscle tone inhibitory system in cats. Arch Ital Biol, 149(4), 385-405. https://doi.org/10.4449/aib.v149i4.1383

Terra, H., Bruinsma, B., de Kloet, S. F., van der Roest, M., Pattij, T., \& Mansvelder, H. D. (2020). Prefrontal Cortical Projection Neurons Targeting Dorsomedial Striatum Control Behavioral Inhibition. Current Biology, 30(21), 4188-4200.e4185. https://doi.org/https://doi.org/10.1016/j.cub.2020.08.031

Treisman, A. M. (1964). Selective attention in man. British medical bulletin, 20(1), 12-16.

Xiong, Q., Znamenskiy, P., \& Zador, A. M. (2015). Selective corticostriatal plasticity during acquisition of an auditory discrimination task. In Nature (Vol. 521, pp. 348-351).

Yin, H. H., Knowlton, B. J., \& Balleine, B. W. (2004). Lesions of dorsolateral striatum preserve outcome expectancy but disrupt habit formation in instrumental learning. European journal of neuroscience, 19(1), 181-189.

Zagha, E. (2020). Shaping the Cortical Landscape: Functions and Mechanisms of Top-Down Cortical Feedback Pathways [Hypothesis and Theory]. Frontiers in Systems Neuroscience, 14. https://doi.org/10.3389/fnsys.2020.00033

Zagha, E., Erlich, J. C., Lee, S., Lur, G., O'Connor, D. H., Steinmetz, N. A., Stringer, C., \& Yang, H. (2022). The Importance of Accounting for Movement When Relating Neuronal Activity to Sensory and Cognitive Processes. J Neurosci, 42(8), 1375-1382. https://doi.org/10.1523/jneurosci.1919-21.2021

Zagha, E., Ge, X., \& McCormick, D. A. (2015). Competing neural ensembles in motor cortex gate goal-directed motor output. Neuron, 88(3), 565-577.

Zareian, B., Zhang, Z., \& Zagha, E. (2021). Cortical Localization of the Sensory-Motor Transformation in a Whisker Detection Task in Mice. Eneuro, 8(1).

Zatka-Haas, P., Steinmetz, N. A., Carandini, M., \& Harris, K. D. (2021). Sensory coding and the causal impact of mouse cortex in a visual decision. elife, 10. https://doi.org/10.7554/eLife.63163 


\section{Figures}

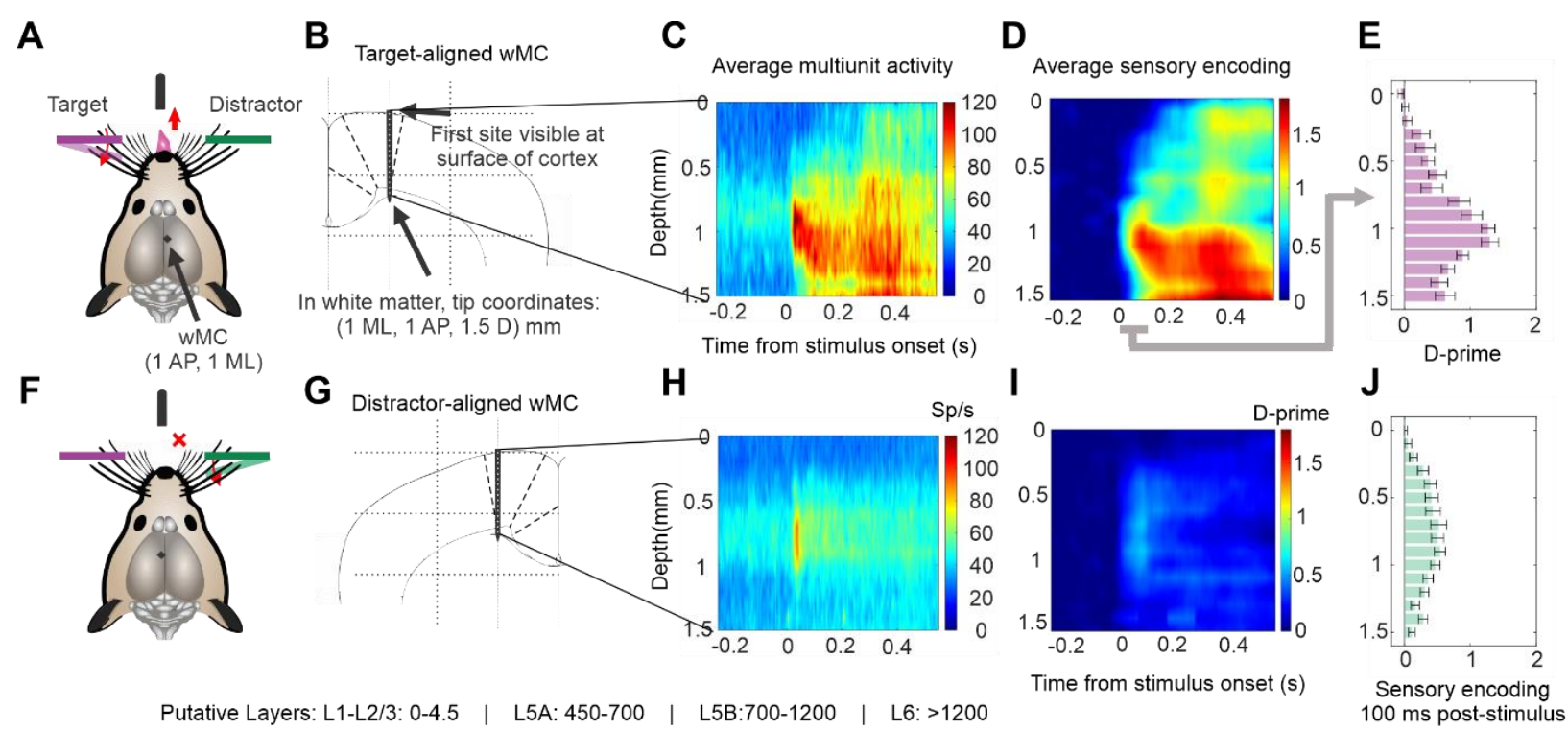

Figure 1. Attenuation of distractor sensory responses in deep layers of whisker motor cortex.

(A) Illustration of the behavioral task and target-aligned wMC recording site. Mice learn to respond (lick) to small, transient whisker deflections in one whisker field (purple - target) and inhibit licking to identical deflections in the opposite whisker field (green - distractor). (B) Schematic of a laminar recording probe in target-aligned wMC, coronal projection. The laminar probes span all cortical layers. (C) Average multiunit activity in target-aligned wMC ( $\mathrm{n}=11$ sessions), aligned to the onset of the target stimulus. The $\mathrm{x}$-axis denotes time from stimulus onset in seconds, the y-axis denotes depth below the pial surface in $\mathrm{mm}$. Multiunit spiking responses to target stimuli occurs throughout the column, yet most robustly in deep layers $(>0.7 \mathrm{~mm}$, putative layer 5B and layer 6). The colormap depicts average multiunit spike rate (Hz). (D) Spatiotemporal distribution of target-aligned wMC sensory encoding, based on the data in [C]. The colormap depicts neurometric d-prime values. (E) Laminar profile of sensory encoding magnitude within the first $100 \mathrm{~ms}$ after the stimulus onset. (F-J) Similar to A to E but for processing of distractor stimuli recorded from the distractor-aligned $\mathrm{wMC}$ ( $\mathrm{n}=19$ sessions). Spiking responses $[\mathrm{H}]$ and stimulus encoding $[\mathrm{I}, \mathrm{J}]$ are smaller amplitude and more transient compared to target stimulus responses in target-aligned wMC. 
A
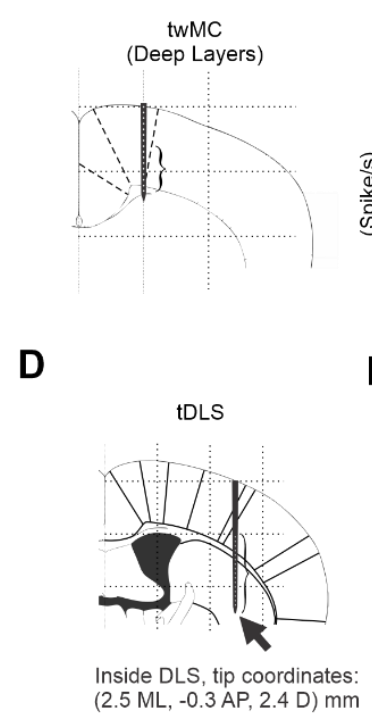

B

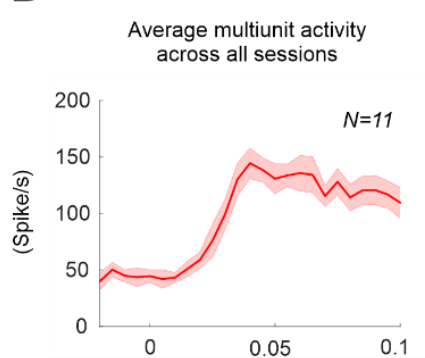

E

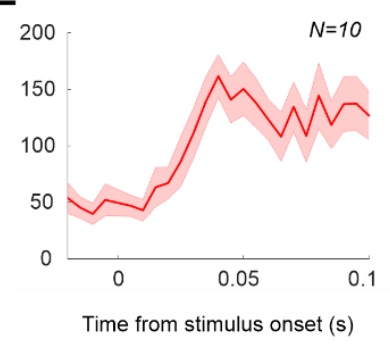

C

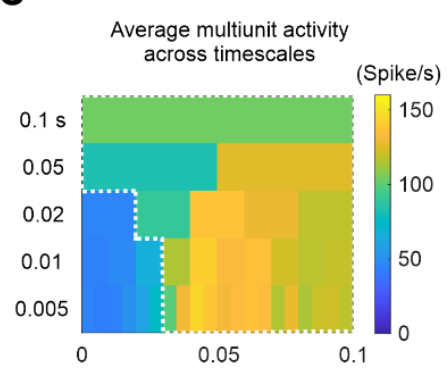

$\mathbf{F}$

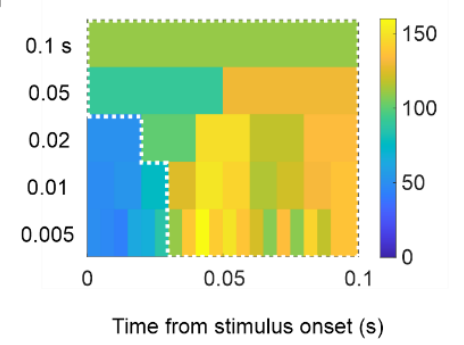

G

Sensory encoding

across deep layers
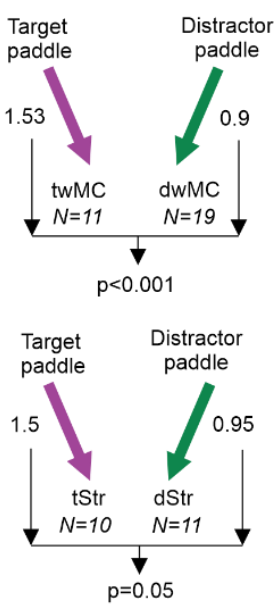

Figure 2. Deep layers of whisker motor cortex and dorsolateral striatum show similar sensory responses and target-distractor attenuation.

(A) Schematic of a laminar recording probe in target-aligned wMC and identification of deep layers, coronal projection. (B) Average peak multiunit activity of target stimulus responses in deep layers of $\mathrm{wMC}(\mathrm{n}=11$ sessions). The $\mathrm{x}$-axis denotes time from stimulus onset in seconds, the $\mathrm{y}$-axis denotes a multiunit spike rate in $\mathrm{Hz}$. Note the rapid increase in spiking activity after stimulus onset. (C) Quantification of spiking activity in [B] for different binning and timescales, starting from $5 \mathrm{~ms}$ non-overlapping bins (bottom) to a single $100 \mathrm{~ms}$ bin (top). Bins significantly above pre-stimulus baseline are indicated by a dashed line (outer area, larger than $2 *$ std of the baseline activity). (D) Schematic of a laminar recording probe in target-aligned dorsolateral striatum (DLS), coronal projection. (E and F) Similar to [B and C] but for target stimulus responses in target-aligned DLS ( $\mathrm{n}=10$ sessions). Note similar temporal response patterns in wMC and DLS. (G) Comparison of target-aligned encoding of target stimuli and distractoraligned encoding of distractor stimuli, across the first $100 \mathrm{~ms}$ post-stimulus. The values next to the arrows denote average sensory encoding (neurometric d-prime) for each condition. Both deep layers of wMC (top) and DLS (bottom) show similar encoding for each condition, and similar reductions in distractor compared to target encoding (attenuation). 
A

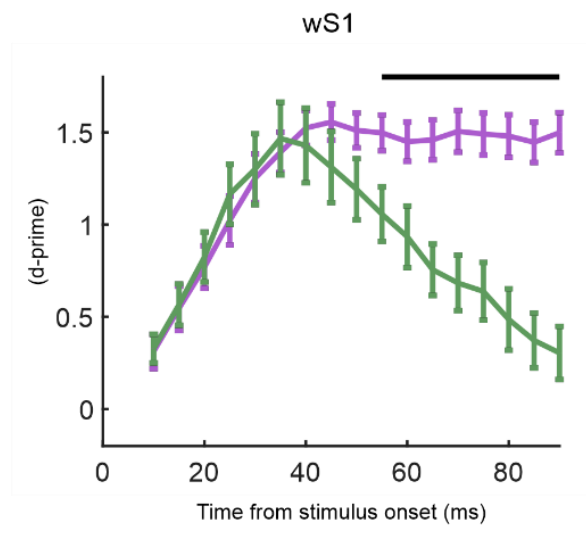

C

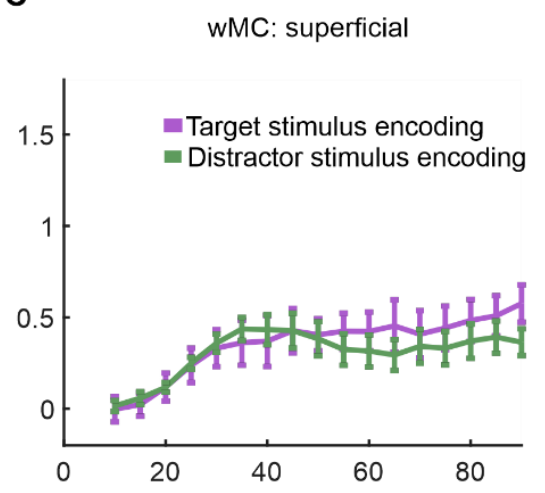

B

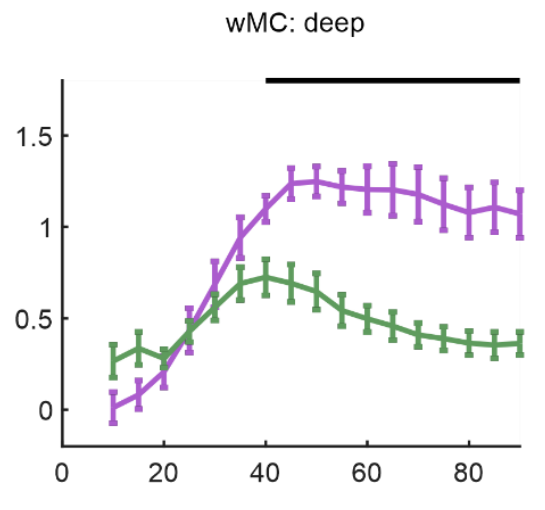

D

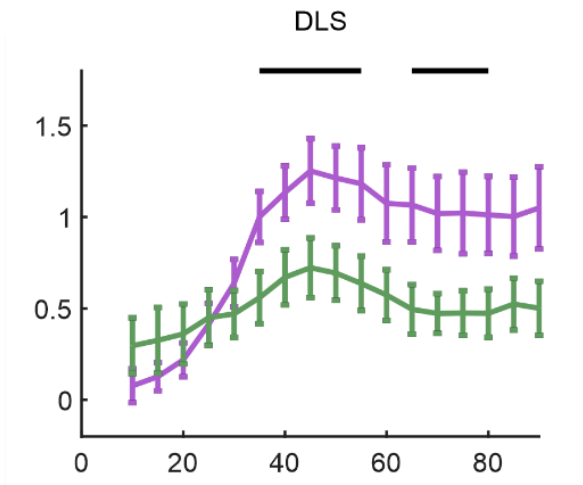

Figure 3. Distractor attenuation occurs in wMC and DLS before wS1.

Time course of distractor attenuation as determined by the earliest significant difference in target and distractor encoding within each region. (A) Average target (purple) and distractor (black) sensory encoding values recorded from layer 5 of wS1, for $20 \mathrm{~ms}$ sliding windows with $75 \%$ overlap. Within wS1, target and distractor encoding diverge after $55 \mathrm{~ms}$ post-stimulus onset (black bars above traces denote statistical significance). (B-D) Similar to [A] but for deep (B) and superficial (C) layers of wMC and DLS (D). Earliest divergence of target and distractor encoding occurs at approximately $40 \mathrm{~ms}$ for both deep layers of wMC and DLS. Superficial layers of wMC do not display distractor attenuation within this temporal window. 

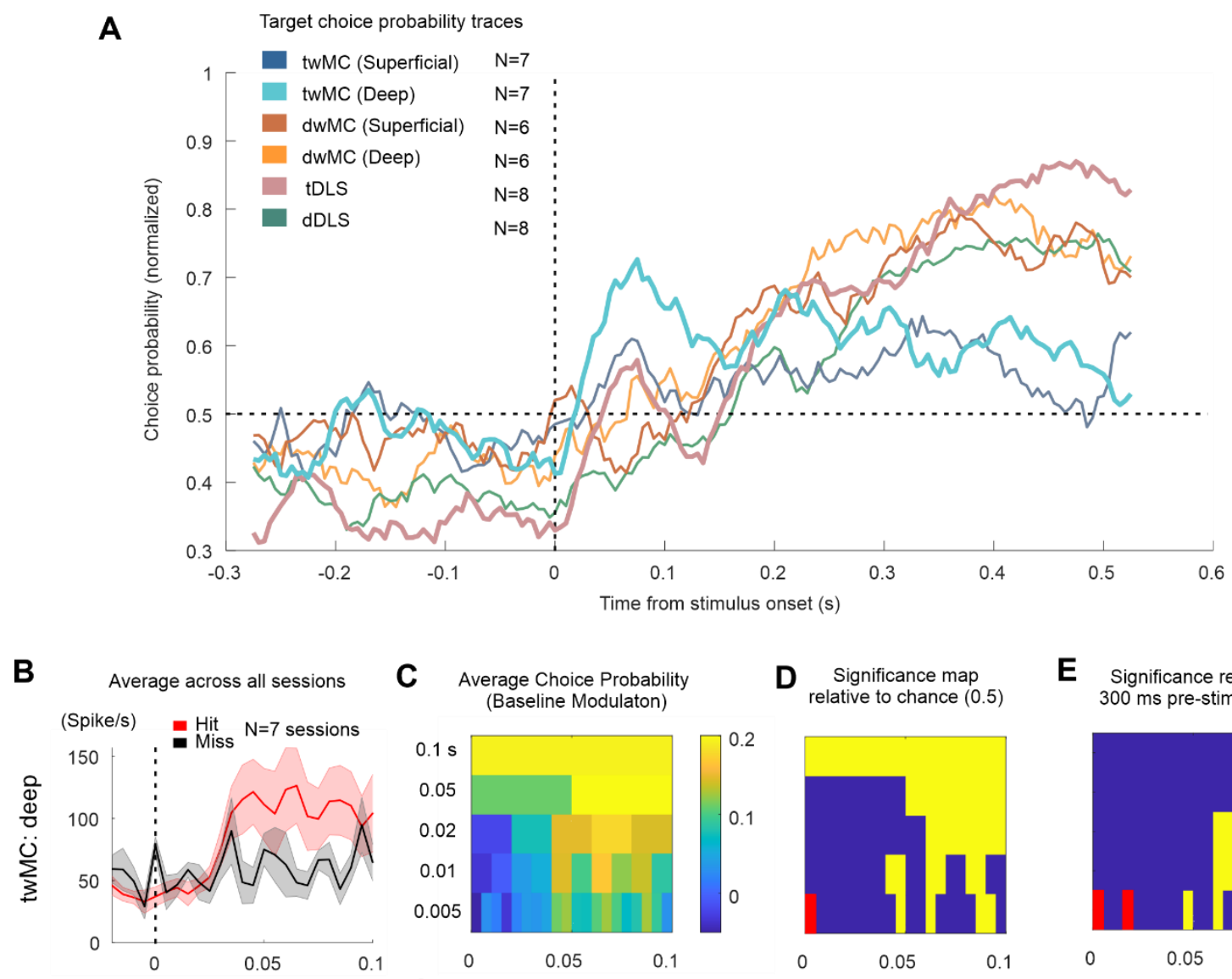

Significance map
relative to chance $(0.5)$

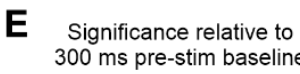
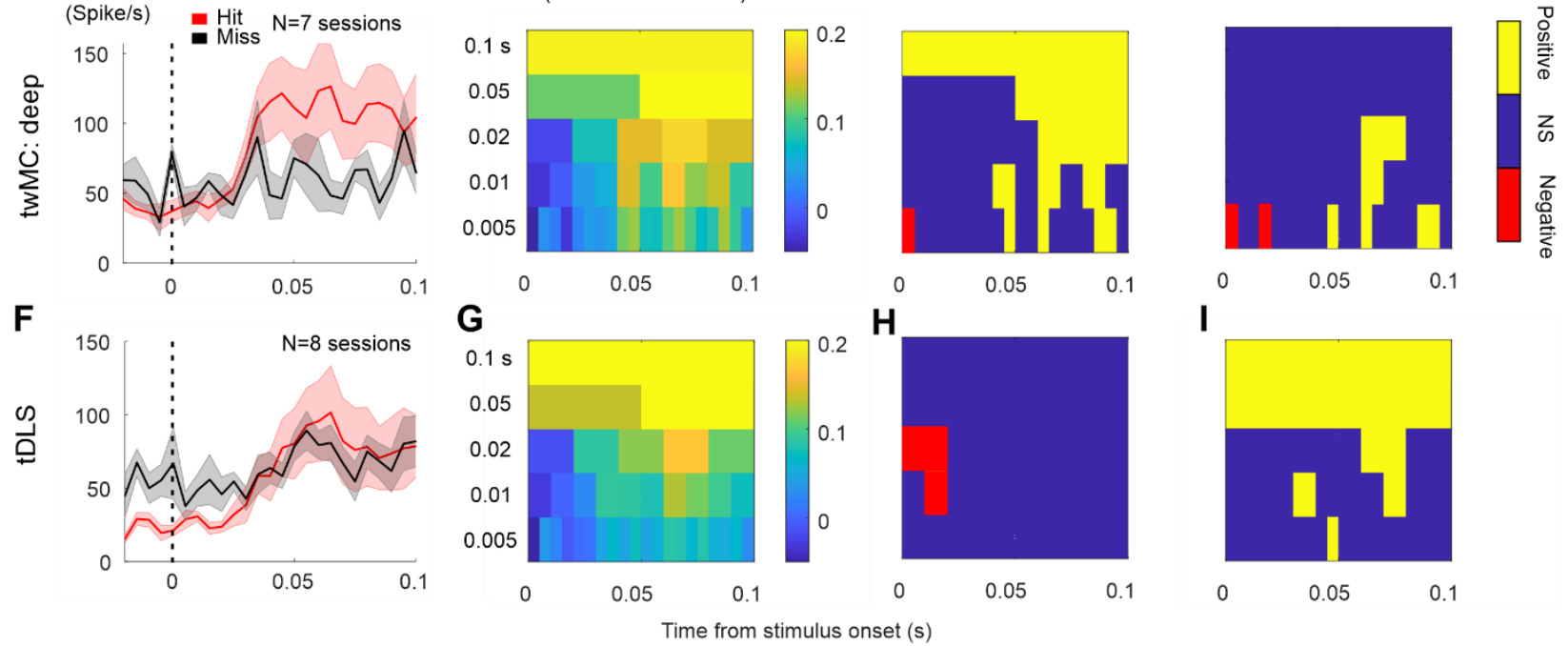

Figure 4. Early and robust choice probability in target-aligned wMC and DLS.

(A) Choice probability traces calculated as the separation of spiking activity on hit and miss trials in 50 ms sliding windows with $90 \%$ overlap. Different colors depict choice probability temporal profiles of different regions. Chance level (equal spiking on hit and miss trials) is depicted by the dashed line at 0.5 . Note the below chance pre-stimulus choice probability for all regions. Target-aligned deep layers of wMC (light blue) and DLS (pink) both display robust rises in choice probability within the first $50 \mathrm{~ms}$ post-stimulus. (B) Average multiunit activities recorded from deep layers of target wMC for hit (red) and miss trials (black). (C) Quantification of choice probability (normalized to pre-stimulus choice probability) based on spiking data in [B] for different binning and timescales, from $5 \mathrm{~ms}$ bins (bottom) a single $100 \mathrm{~ms}$ bin (top). (D) Significance testing of post-stimulus choice probability relative to chance level (0.5), with the same binning format as in [C]. Bins with significant below chance choice probability are depicted in red; bins with significant above chance choice probability are depicted in yellow. (E) Significance testing of post-stimulus choice probability relative to pre-stimulus choice probability, with the same binning format as in [C] and [D]. Significant above chance choice 
bioRxiv preprint doi: https://doi.org/10.1101/2022.03.03.482906; this version posted March 4, 2022. The copyright holder for this preprint (which was not certified by peer review) is the author/funder, who has granted bioRxiv a license to display the preprint in perpetuity. It is made available under aCC-BY-NC 4.0 International license.

probability in wMC emerges within 45-70 ms post-stimulus. (F-I) Similar to [B-E] but for DLS. Significant above chance choice probability in DLS emerges within 40-80 ms post-stimulus, yet only when referenced to pre-stimulus levels (I). 

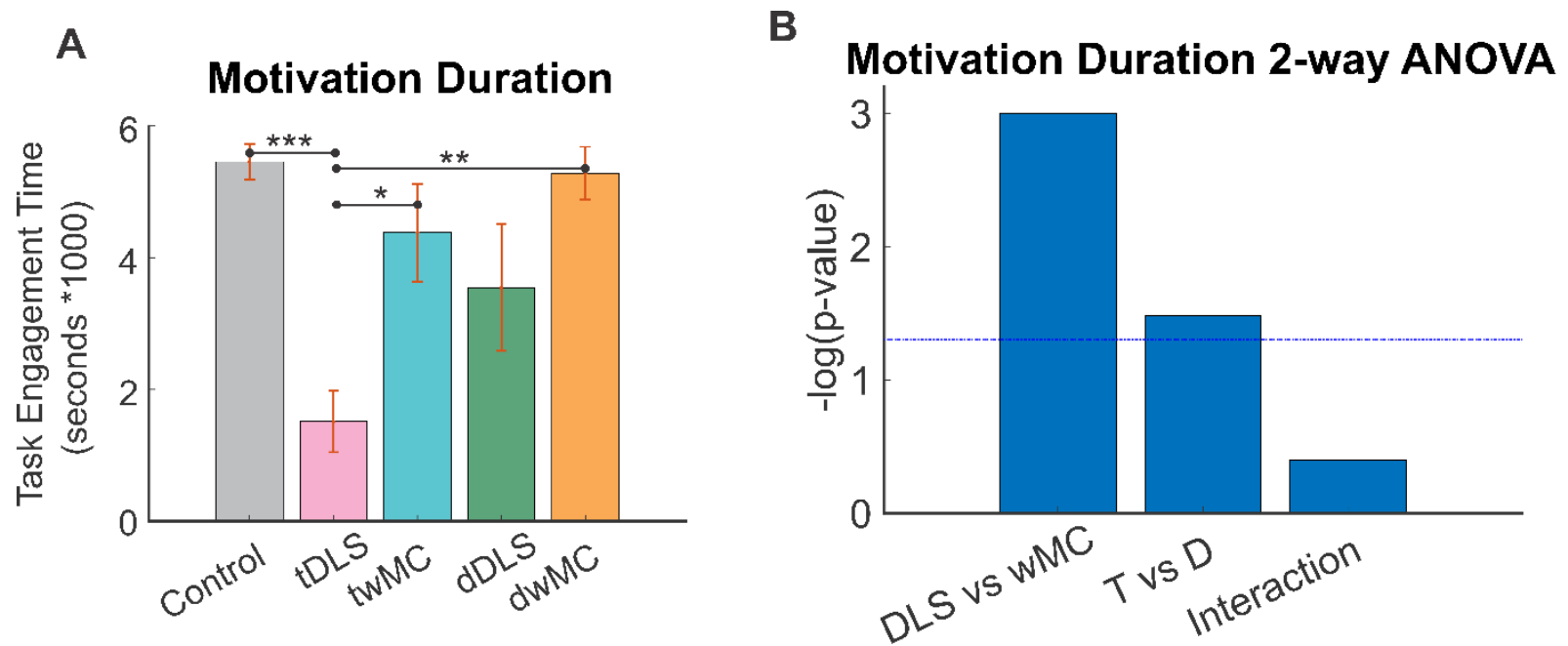

Figure 5. Target-aligned DLS inactivation reduces task engagement.

(A) Length of task engagement (motivation duration) as defined by the persistence of licking responses, averaged across all sessions according to the region of inactivation (control $n=72$ sessions, tDLS $\mathrm{n}=10$ sessions, twMC $\mathrm{n}=12$ sessions, $\mathrm{dDLS} \mathrm{n}=10$ sessions, $\mathrm{dwMC} \mathrm{n}=13$ sessions) (tDLS, target-aligned DLS; twMC, target-aligned wMC; dDLS, distractor-aligned DLS; dwMC, distractor-aligned wMC). Lines connecting individual bar plots reflect statistical significance from post-hoc pair-wise comparisons. $* \mathrm{p}<05$; ** $\mathrm{p}<01$; *** $\mathrm{p}<001$. Note the significant reduction in motivation duration for tDLS compared to control sessions. (B) Results from a 2way ANOVA analysis comparing main effects of region (DLS versus wMC inactivation) and hemisphere (target-aligned versus distractor-aligned inactivation) and interaction effects. Dotted line reflects threshold for statistical significance $(\mathrm{p}=0.05)$, with larger values on the $y$-axis reflecting smaller p-values. This plot shows significant main effects of region and hemisphere, but not their interaction. Similar data displays as in $[\mathrm{A}]$ and $[\mathrm{B}]$ are used in figure 6 and supplemental figures 3 and 4 . 

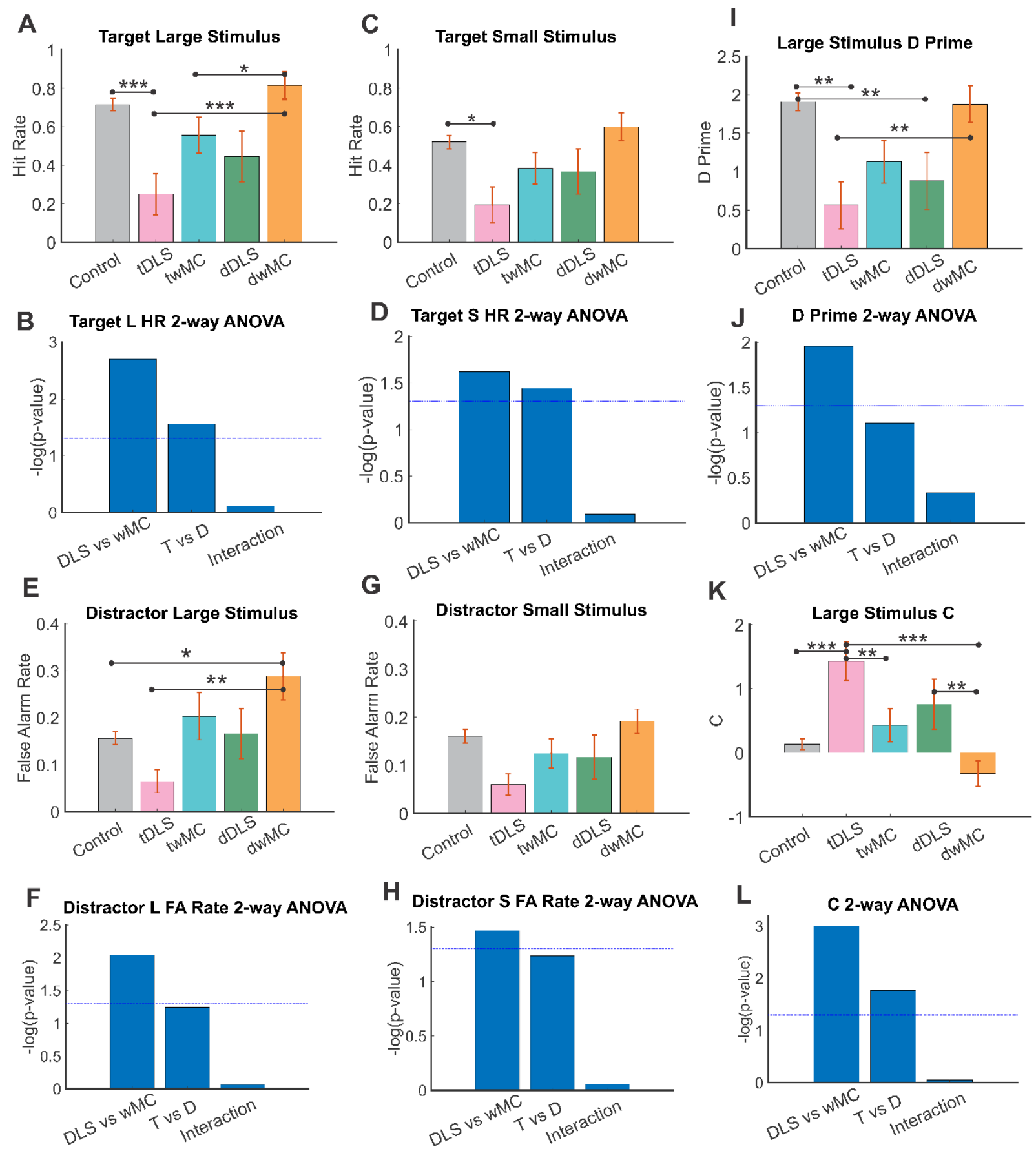

Figure 6. Effects of DLS and wMC inactivation on multiple measures of task performance. Same conventions as in figure 5, comparing results from one-way and two-way ANOVA analyses. Shown are analyses of large hit rate $(A, B)$, small hit rate $(C, D)$, large false alarm rate $(\mathrm{E}, \mathrm{F})$, small false alarm rate $(\mathrm{G}, \mathrm{H})$, large stimulus discrimination d-prime $(\mathrm{I}, \mathrm{J})$, and large stimulus criterion $(\mathrm{C})(\mathrm{K}, \mathrm{L})$. For target-aligned DLS inactivation, note significant reductions in response rates and d-prime and increases in criterion. For distractor-aligned wMC, note increases in response rates, in particular large false alarm rate, and reductions in criterion. 
A

\section{Target Hemisphere}

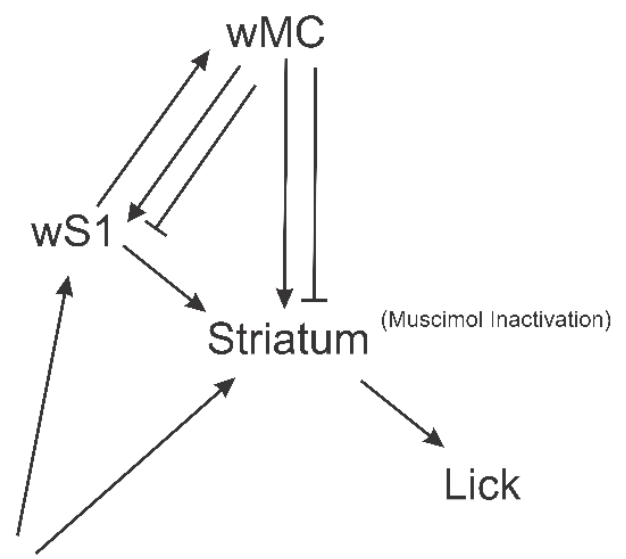

Target Stimulus
B

\section{Distractor Hemisphere}

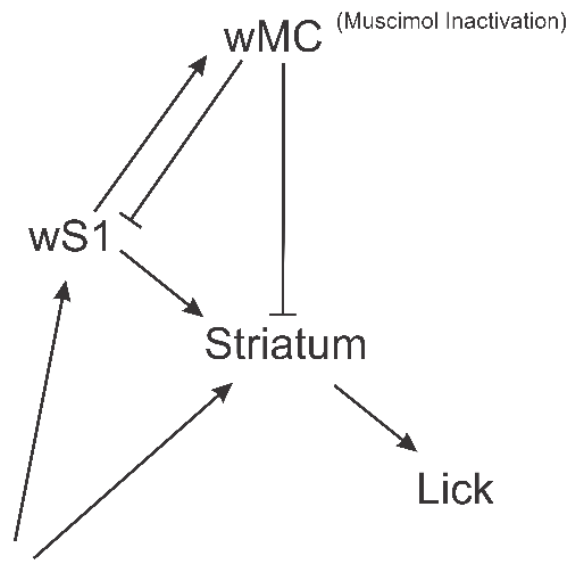

Distractor Stimulus

Figure 7. Circuit model for how wMC and DLS may interact to implement sensory selection and inhibition, based on our recording and inactivation data.

(A) Target-aligned DLS functions as a critical node to produce "go" behavior, potentially integrating sensory evidence from wS1, wMC, and subcortical inputs. (B) In contrast to DLS, distractor-aligned wMC plays essential roles in preventing "no-go" behavior. This may occur by suppressing distractor stimulus responses in wS1 and/or DLS. 
Supplemental Figures

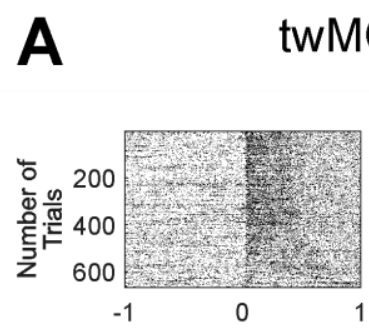

: deep

B

\section{tDLS}
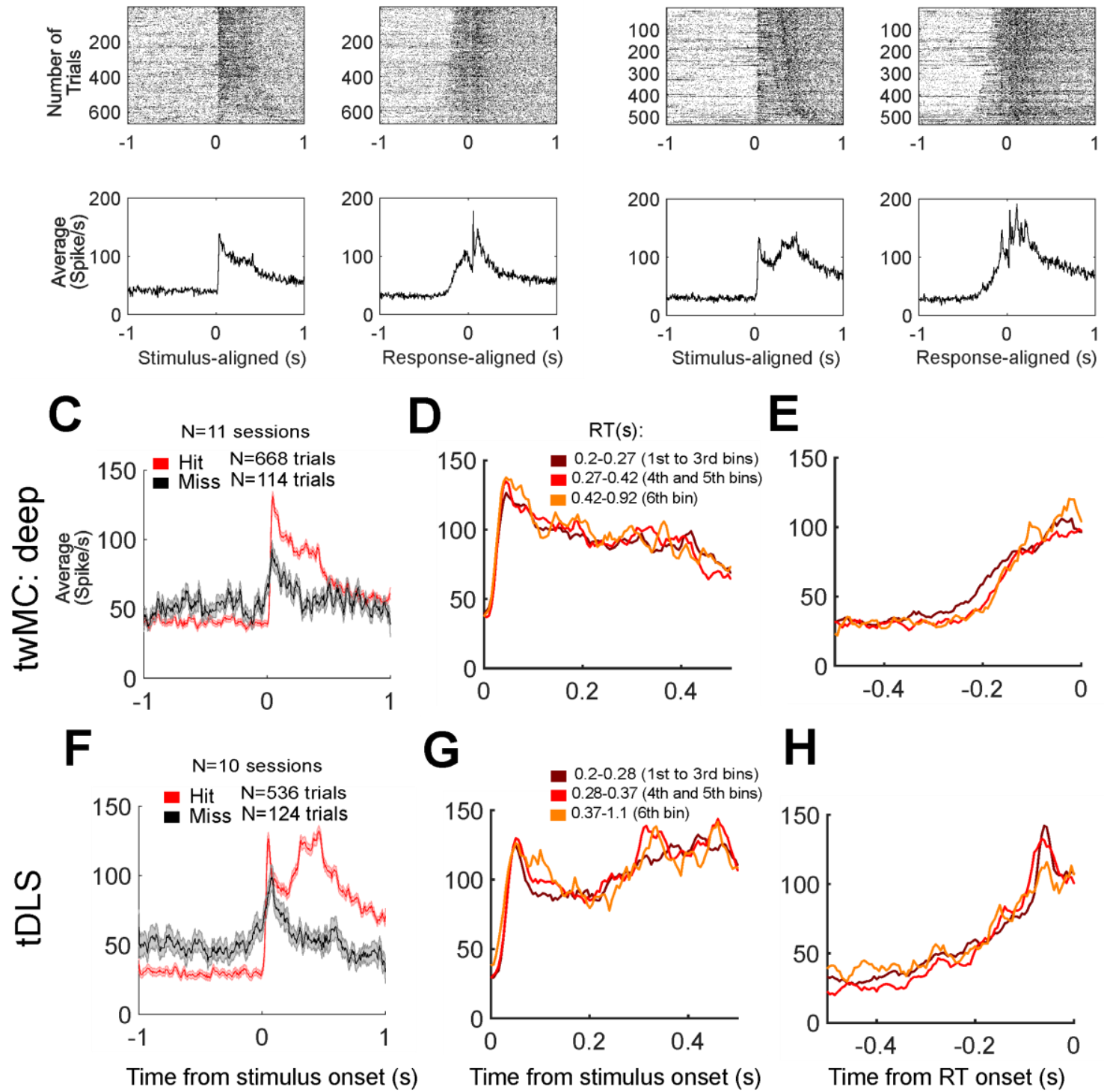

Supplemental Figure 1. Similar sensory and motor representations in target-aligned wMC and DLS

(A) Multiunit activity recorded from deep layers of target-aligned wMC for all hit trials pooled across sessions. The left column depicts alignment to the target stimulus onset and the right column depicts alignment to the reaction time in seconds. Top panels show raster plots sorted based on reaction times from fast (top) to slow (bottom). Bottom panels show average of all trials shown in the top panels. Note the rapid post-stimulus (left, putative sensory) and the robust pre-response (right, putative motor) spiking activities. (B) Same as [A] but recorded from target aligned DLS. (C) Average of all target stimulus hit (red) and miss (black) trials recorded from deep layers of target-aligned wMC ( $\mathrm{n}=11$ sessions). (D) Target stimulus hit trials from targetaligned wMC grouped based on reaction time, aligned to the stimulus onset. Darker colors depict 
faster reaction time trials. Note the early 'sensory' peak, invariant to reaction time. (E) Target stimulus hit trials from target-aligned wMC grouped based on reaction time (as in [D]), aligned to the reaction time. Note the ramping 'motor' signals, achieving similar activity levels before the reaction time. (F, G and H) Same as [C, D and E] but recorded from target-aligned DLS, also displaying robust sensory and motor alignments. 
A
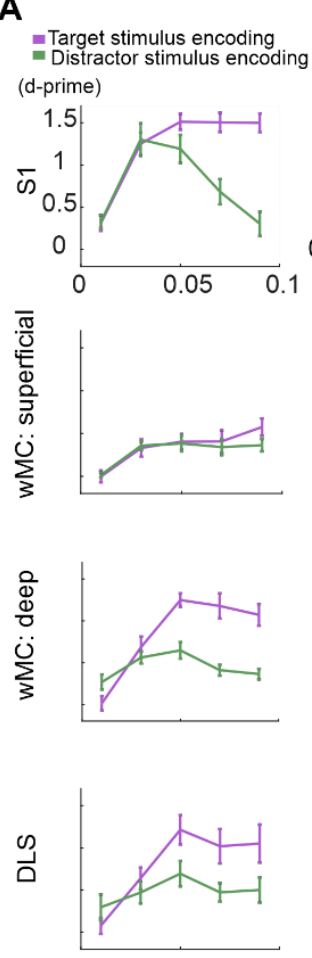

Time from stimulus onset $(\mathrm{s})$
B
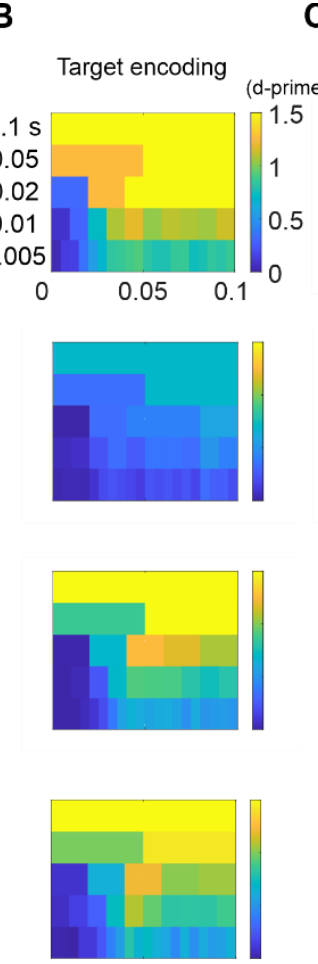

$$
\text { (5) }
$$

C
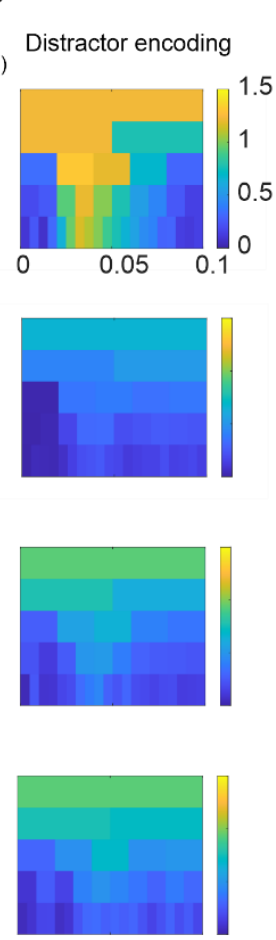

Time from stimulus onset (s)

D Modulation index
$(T-D) /(T+D)$
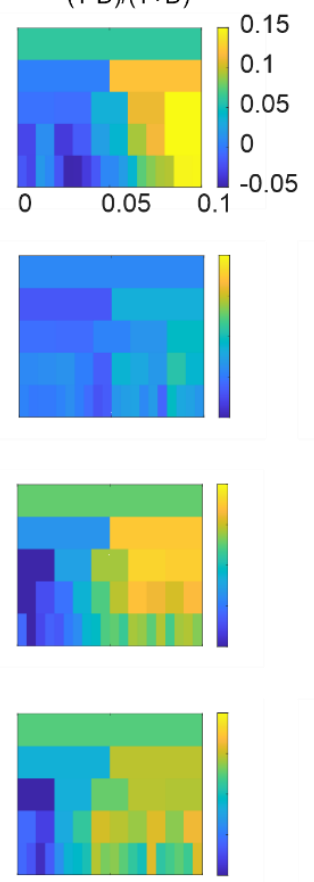

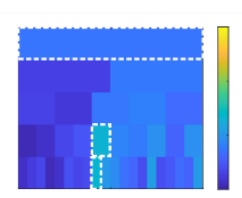

E
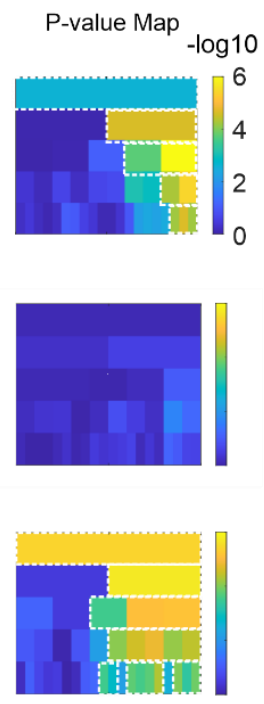

\section{Supplemental Figure 2. Distractor stimulus attenuation calculated for different binning and timescales.}

(A) Average sensory encoding values across sessions, for $20 \mathrm{~ms}$ non-overlapping windows in wS1, superficial wMC, deep wMC and DLS. Purple color depicts target sensory encoding from the target-aligned hemisphere and green color depicts distractor sensory encoding from the distractor-aligned hemisphere. (B) Target sensory encoding averaged across sessions, for different binning and timescales starting from $5 \mathrm{~ms}$ non-overlapping bins (bottom) to a single $100 \mathrm{~ms}$ window (top). (C) Distractor sensory encoding averaged across sessions, with the same binning and timescales as in [B]. (D) Modulation index (divergence) calculated from the target and distractor sensory encoding profiles in [B and C]. (E) P-value profiles for data in [D]. Significant regions are marked by dashed lines (two-sample t test, Bonferroni-corrected for each timescale, alpha=0.05). 
A

\section{Target Large Stimulus: engaged period}

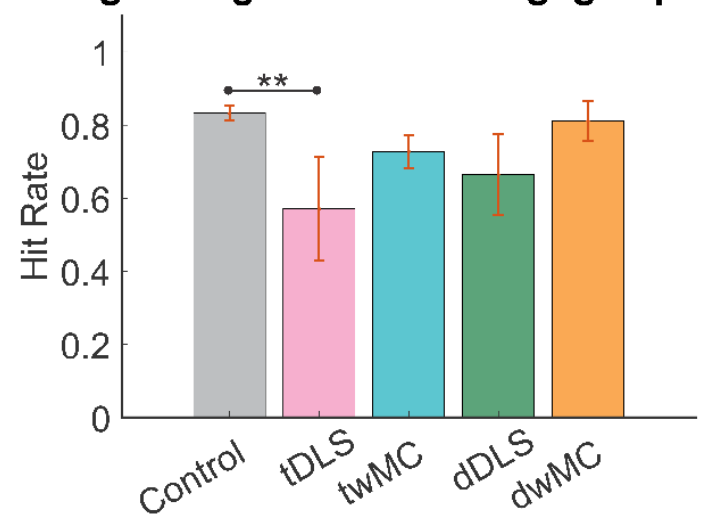

B

Distractor Large Stimulus: engaged period

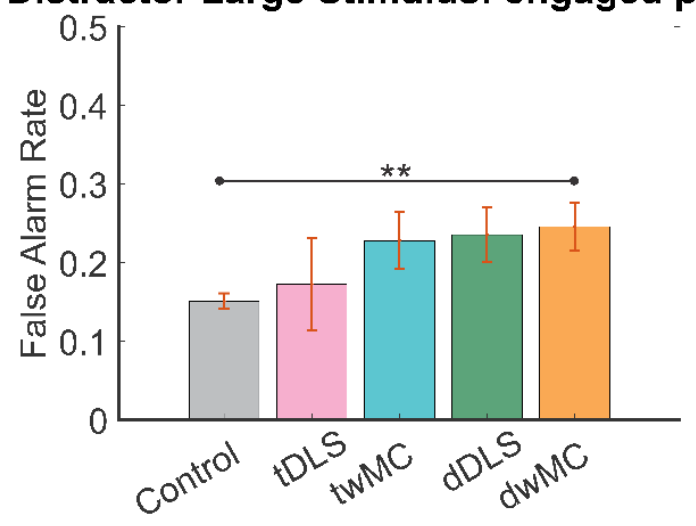

Supplemental Figure 3. Effects of inactivation on large stimulus hit and false alarm rates, when only considering temporal windows with high engagement.

Active engagement was determined as periods of at least 5 minutes with no lapses in licking greater than 60 seconds. (A) Hit rates for large target stimuli averaged across all sessions, according to the region of inactivation. Note, reduced hit rates for target-aligned DLS (tDLS) inactivation, even during periods of high task engagement. (B) False alarm rates for large distractor stimuli averaged across all sessions, according to the region of inactivation. Note, increased false alarm rates for distractor-aligned wMC (dwMC) inactivation. 
A

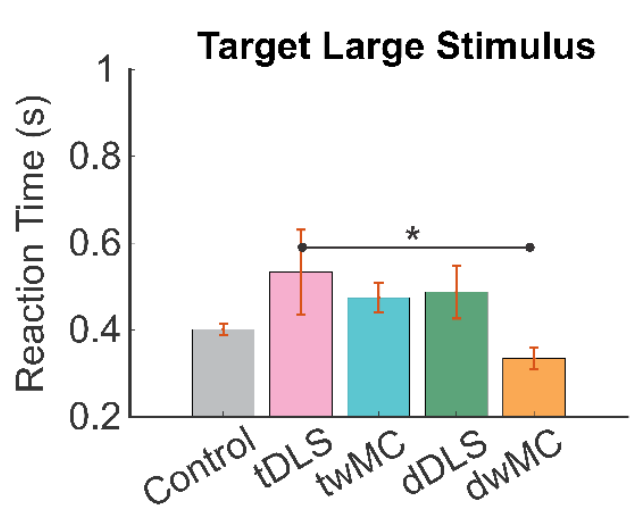

C

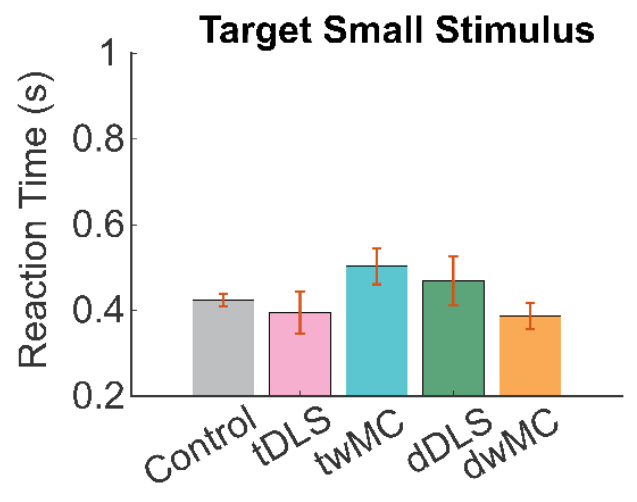

E

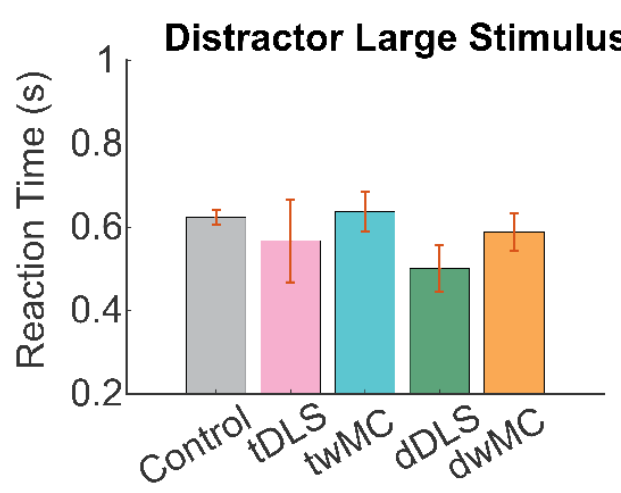

G

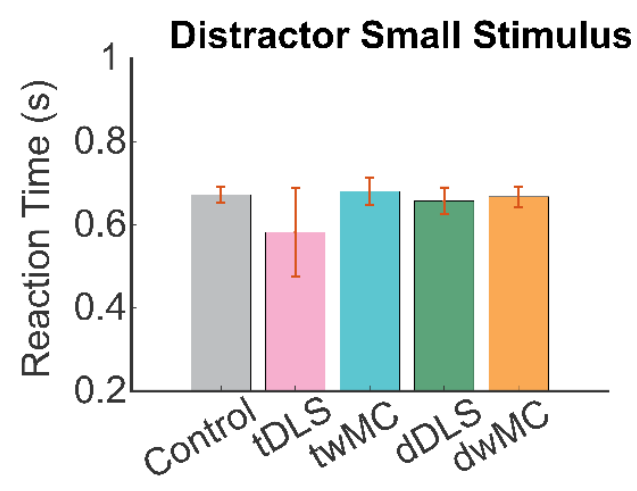

B

Target Large Stimulus Reaction Time 2-way ANOVA

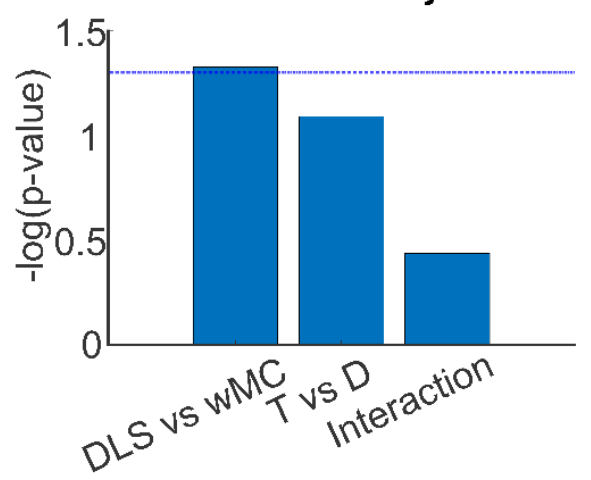

Target Small Stimulus

D

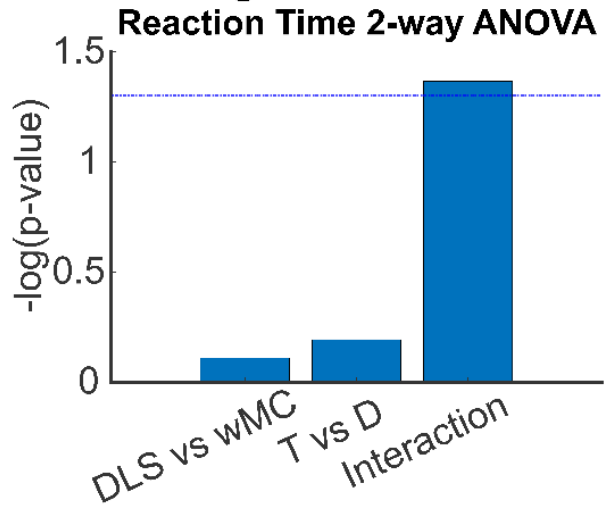

F

Distractor Large Stimulus

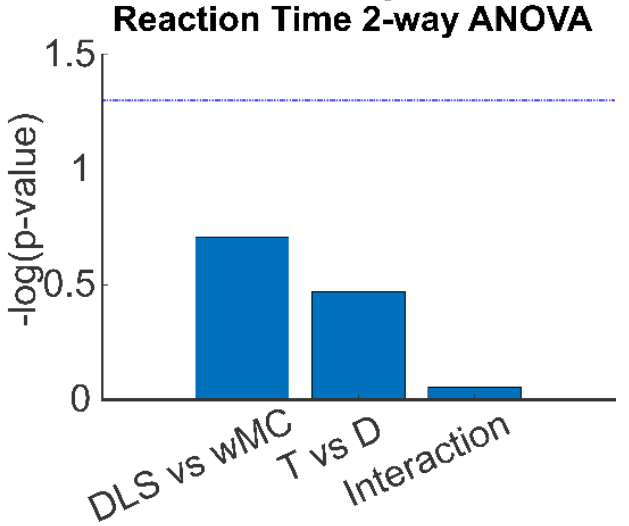

Distractor Small Stimulus

Reaction Time 2-way ANOVA

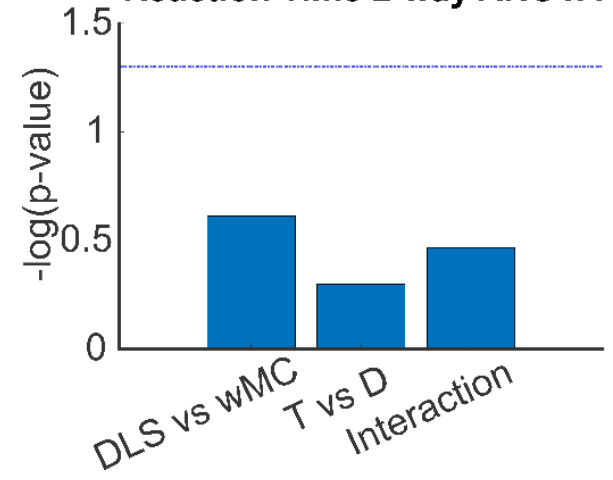




\section{Supplemental 4. Reaction time is unchanged from control sessions for all regions of inactivation.}

$(A, C)$ Hit reaction times for large (A) and small (C) stimuli strengths, separated by region of inactivation. (E,G) False alarm reaction times for large $(\mathrm{E})$ and small $(\mathrm{G})$ stimuli strengths, separated by region of inactivation. (B,D,F,H) 2-way ANOVA analyses, comparing main effects of region (DLS versus wMC inactivation) and hemisphere (target-aligned versus distractoraligned inactivation) and their interactions. Dotted line reflects threshold for statistical significance $(\mathrm{p}=0.05)$, with larger values on the $y$-axis reflecting smaller $p$-values. 


\section{Behavior Workflow}

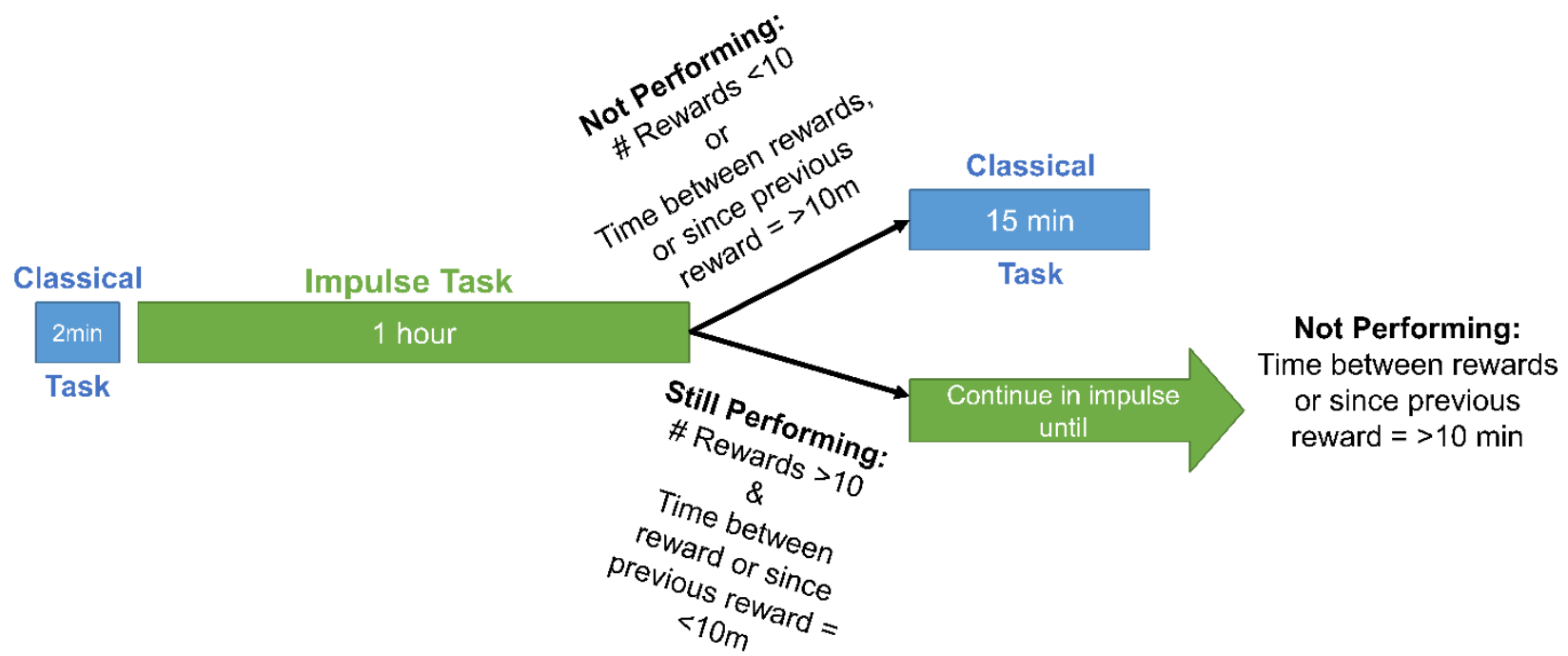

\section{Supplemental Figure 5. Behavioral training workflow diagram for muscimol inactivation experiments. \\ For each behavioral training day, mice are placed in a classical conditioning task for 2 minutes} ('Classical 1' in Supplemental Figure 7), followed by the impulse control task for at least 1 hour. Continuation in the impulse task is allowed for mice still performing the task (gained $>10$ rewards and the time since previous reward is less than 10 minutes). For mice no longer performing the impulse task $(<10$ rewards or at least 10 minutes has passed since previous reward), they are placed back in a classical conditioning task ('Classical 2' in Supplemental Figure 7) for 15 minutes (see also methods). 

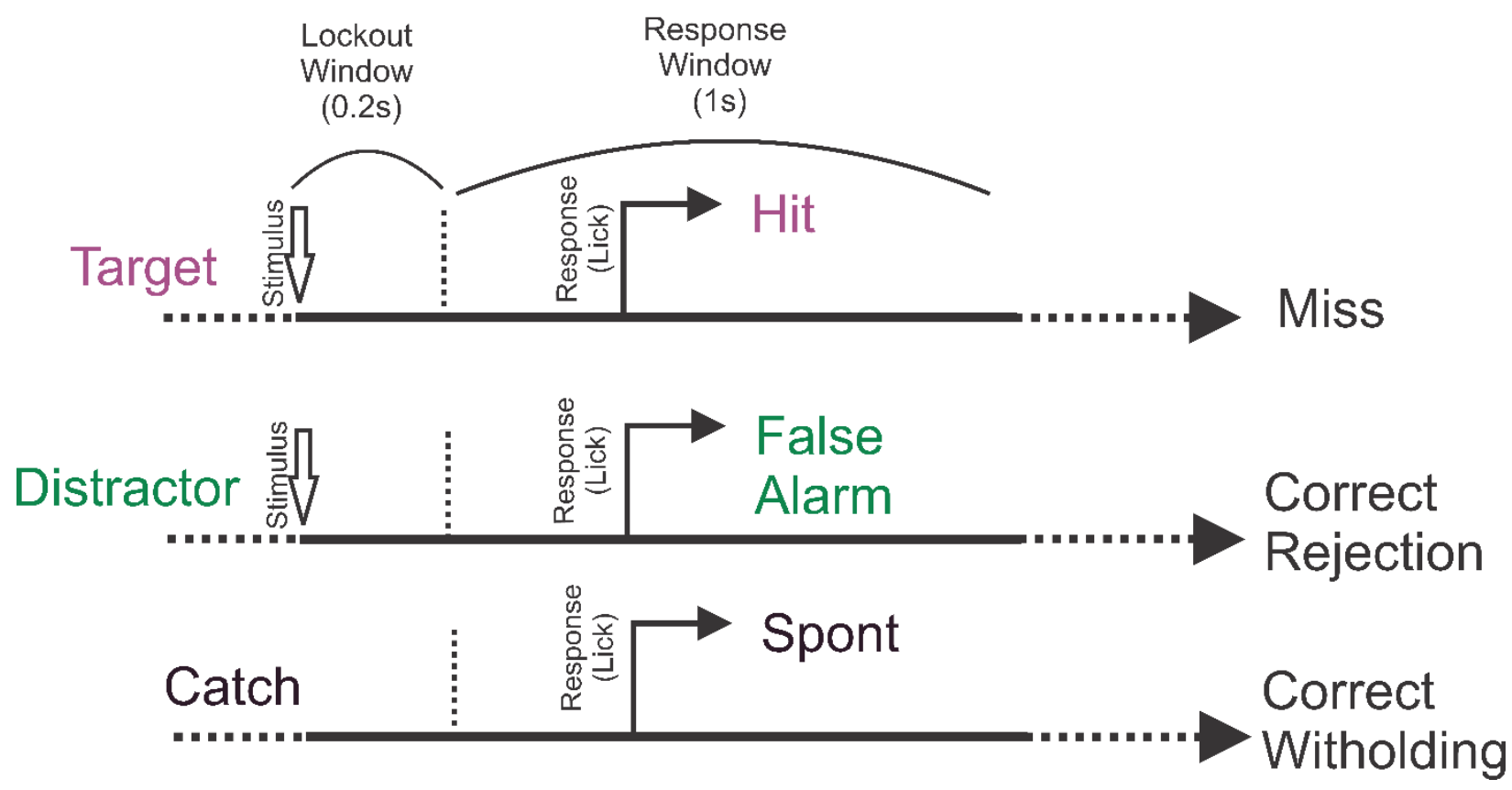

\section{Supplemental Figure 6. Full selective detection task structure.}

Diagram of task structure for the full impulse control version of the selective detection task (also see methods). Following a variable (5-9s) intertrial interval, mice receive either a target, distractor or catch trial. After stimulus onset, a lockout window of $0.2 \mathrm{~s}$ is implemented in which responding is punished by immediately restarting the intertrial (effectively, a time out). Responses during the response window of target trials are considered hits and responses during the response window of distractor trials are considered false alarms. 

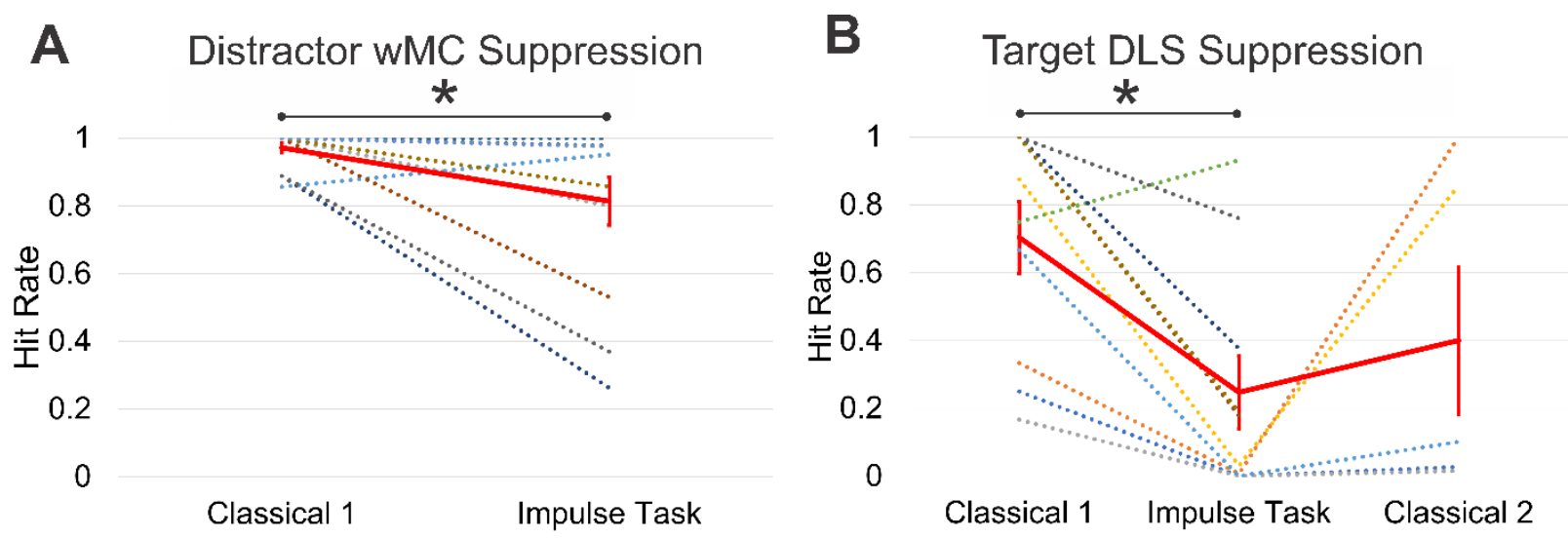

\section{Supplemental Figure 7. Muscimol inactivation of target-aligned DLS does not impair the ability to respond.}

(A) Hit rates during distractor-aligned wMC inactivation (shown are individual sessions in dotted lines and their average in red) for the classical conditioning task 1 (2min, left) and the impulse control task (1-hour, right). 13 out of 13 sessions had above $50 \%$ hit rates in classical conditioning task 1, with no sessions meeting criteria for training in a second classical conditioning phase. (B) Same as [A], but for target-aligned DLS inactivation sessions, and including data from classical conditioning task 2 . For target-aligned DLS inactivation, 8 out of 10 sessions had above $50 \%$ hit rates in at least 1 classical conditioning phase. 